\title{
RASS-SDSS Galaxy clusters survey
}

\section{The catalog and the correlation of X-ray and optical properties ${ }^{\star}$}

\author{
P. Popesso ${ }^{1}$, H. Böhringer ${ }^{1}$, J. Brinkmann ${ }^{2}$, W. Voges ${ }^{1}$, and D. G. York ${ }^{3}$ \\ 1 Max-Planck-Institut fur extraterrestrische Physik, 85748 Garching, Germany \\ e-mail: popesso@mpe.mpg.de \\ 2 Apache Point Observatory, PO Box 59, Sunspot, NM 88349, USA \\ 3 Department of Astronomy and Astrophysics, University of Chicago, 5640 South Ellis Avenue, Chicago, IL 60637, USA
}

Received 8 December 2003 / Accepted 4 March 2004

\begin{abstract}
For a detailed comparison of the appearance of cluster of galaxies in X-rays and in the optical, we have compiled a comprehensive database of X-ray and optical properties of a sample of clusters based on the largest available X-ray and optical surveys: the ROSAT All Sky Survey (RASS) and the Sloan Digital Sky Survey (SDSS). The X-ray galaxy clusters of this RASS-SDSS catalog cover a wide range of masses, from groups of $10^{12.5} M_{\odot}$ to massive clusters of $10^{15} M_{\odot}$ in the redshift range 0.002-0.45. The RASS-SDSS sample comprises all the X-ray selected objects already observed by the Sloan Digital Sky Survey (114 clusters). For each system we have uniformly determined the X-ray (luminosity in the ROSAT band, bolometric luminosity, center coordinates) and optical properties (Schechter luminosity function parameters, luminosity, central galaxy density, core, total and half-light radii). For a subsample of 53 clusters we have also compiled the temperatures and the iron abundances from the literature. The total optical luminosity can be determined with a typical uncertainty of $20 \%$ independent of the choice of local or global background subtraction. We searched for parameters which provide the best correlation between the X-ray luminosity and the optical properties and found that the $z$ band luminosity determined within a cluster aperture of $0.5 \mathrm{Mpc} h_{70}^{-1}$ provides the best correlation, with a scatter of about $60-70 \%$. The scatter decreases to less than $40 \%$ if the correlation is limited to the bright X-ray clusters. The resulting correlation of $L_{\mathrm{X}}$ and $L_{\mathrm{op}}$ in the $z$ and $i$ bands shows a logarithmic slope of 0.38 , a value not consistent with the assumption of a constant $M / L$. Consistency is found, however, for an $M / L$ increasing with luminosity as suggested by other observations. We also investigated the correlation between $L_{\mathrm{op}}$ and the X-ray temperature, obtaining the same result.
\end{abstract}

Key words. galaxies: clusters: general - galaxies: luminosity function, mass function - methods: observational

\section{Introduction}

Clusters of galaxies are the largest well defined building blocks of our Universe. They form via gravitational collapse of cosmic matter over a region of several megaparsecs. Cosmic baryons, which represent approximately $10-15 \%$ of the mass content of the Universe, follow dynamically the dominant dark matter during the collapse. As a result of adiabatic compression and of shocks generated by supersonic motions, a thin hot gas permeates the cluster gravitational potential. For a typical cluster mass of $10^{14} M_{\odot}$ the intracluster gas reaches a temperature of the order of $10^{7} \mathrm{keV}$ and, thus, radiates optically thin thermal bremsstrahlung and line radiation in the X-ray band. In 1978, the launch of the first X-ray imaging telescope, the Einstein observatory, began a new era of cluster

^ Full Tables 1-3 are only available in electronic form at the CDS via anonymous ftp to cdsarc.u-strasbg.fr (130.79.128.5) or via

http://cdsweb.u-strasbg.fr/cgi-bin/qcat?J/A+A/423/449 discovery, as clusters proved to be luminous $\left(\geq 10^{42-45} \mathrm{erg} \mathrm{s}^{-1}\right)$, extended $(r \sim 1-5 \mathrm{Mpc}) \mathrm{X}$-ray sources, readily identified in the X-ray sky. Therefore, X-ray observations of galaxy clusters provide an efficient and physically motivated method of identification of these structures. The X-ray selection is more robust against contamination along the line of sight than traditional optical methods since the richest clusters are relatively rare and since X-ray emission, which is proportional to the gas density squared, is far more sensitive to physical overdensities than the projected number density of galaxies in the sky. In fact the existence of diffuse, very hot X-ray emitting gas implies the existence of a massive confining dark matter halo. Moreover, selection according to X-ray luminosity is also an efficient way to find the highest mass concentrations due to well defined correlation between the X-ray luminosity and the total cluster mass (Reiprich \& Bhöringher 2002). In addition to allowing the identification of galaxy clusters, X-ray observations provide a wealth of information on the intracluster medium itself, e.g. its metal abundance, radial density distribution and 
temperature profile. These latter quantities, in turn, can be used to reliably estimate the total mass of the system.

In addition to the hot, diffuse component, baryons are also concentrated in the individual galaxies within the cluster. These are best studied through photometric and spectroscopic optical surveys, which provide essential information about luminosity, morphology, stellar population and age. Solid observational evidence indicates a strong interaction between the two baryonic components, as galaxies pollute the intracluster medium expelling metals via galactic winds producing the observed metal abundances in clusters (De Grandi et al. 2002; Finoguenov et al. 2001). On the other hand, the evolution of galaxies in clusters is influenced by processes due to the hot gas (e.g. gas stripping by ram pressure, etc.) and also by internal processes like star formation, galactic winds, supernovae explosions etc., operating inside the galaxies themselves (Dressler et al. 1997; Fasano et al. 2000; van Dokkum et al. 2000; Lubin et al. 2002; Kelson et al. 1997, 2000; Ziegler \& Bender 1997; Gomez et al. 2003). In conclusion, understanding the complex physics at play in clusters of galaxies requires combined X-ray and optical observation of a statistically significant sample of these objects.

On the basis of these considerations, we have created a large database of clusters of galaxies based on the largest available X-ray and optical surveys: the ROSAT All Sky Survey (RASS), the only X-ray all sky survey ever realized using an imaging X-ray telescope, and the Sloan Digital Sky Survey (SDSS), which the whole Northern Galactic Cap and part of the Southern Galactic Cap is observed in five wide optical bands covering the entire optical range. By carefully combining the data of the two surveys we have created the RASS-SDSS galaxy cluster catalog. Although two galaxy cluster catalogs from the SDSS already exist, the Cut and Enhance Galaxy Cluster Catalog of Goto et al. (2002a) and the Merged Cluster Catalog of Bahcall et al. (2003, see also Kim et al. 2002), we preferred to compile a new cluster catalog by selecting the systems in the X-ray band for which we have reliable X-ray characteristics, for the reasons explained above. The $\mathrm{X}$-ray-selected galaxy clusters cover a wide range of masses, from groups of $10^{12.5} M_{\odot}$ to massive clusters of $10^{15} M_{\odot}$ in a redshift range from 0.002 to 0.45 . The RASS-SDSS sample comprises all the X-ray detected objects already observed in the sky region covered by the Sloan Digital Sky Survey.

One of the first goals is the comparison of the X-ray and the optical appearance of the clusters. We want in particular find optical parameters that provide the closest correlation to the X-ray parameters, such that we can predict within narrow uncertainty limits the X-ray luminosity from these optical parameters and vice versa. So far optical and X-ray cluster surveys have been conducted independently without much intercomparison. Therefore, the empirical relationship between the $\mathrm{X}$-ray luminosity and optical luminosity of clusters is not so well defined, in large part because of the difficulties inherent in measuring the cluster optical luminosity and in getting a homogeneous set of total optical luminosities for a large number of clusters. Edge \& Stewart (1991) found that the bolometric X-ray luminosity of a local sample of X-ray-selected clusters correlated very roughly with Abell number and somewhat better with the Bahcall galaxy density (number of bright galaxies within $0.5 h^{-1} \mathrm{Mpc}$; Bahcall 1977, 1981) for the small subsample that had Bahcall galaxy densities. Arnaud et al. (1992) made an heroic effort in computing cluster optical luminosities at low redshift from a heterogenous literature. The first joint X-ray/optical survey of galaxy clusters was the ROSAT Optical X-ray Survey (ROXS, Donahue et al. 2002). They observed 23 ROSAT pointings for a total of 5 square degrees in the $I$ band and partially in the $V$ band. The X-ray selection and the optical selection of cluster candidates were done independently, with the wavelets algorithm in the former, and with a matched filter algorithm in the latter case. They found $\mathrm{X}$-ray and optical coincident detections for 26 galaxy clusters. Donahue et al. (2001) studied the relation between the X-ray luminosity and the matched filter parameter $\Lambda_{\mathrm{cl}}$, which is approximately equivalent to the number of $L^{*}$ galaxies in the system (Postman et al. 1998). They found a marginally significant correlation between the two quantities with a significant scatter. Yee \& Ellingson (2003) defined a new richness parameter as the number of cluster galaxies within some fixed aperture, scaled by a luminosity function and a spatial distribution function. They analysed a sample of 15 clusters from the CNOC1 Cluster Redshift Survey, and found a very poor correlation between this parameter and other cluster properties such as the $\mathrm{X}$-ray luminosity, temperature and the velocity dispersion.

In the present paper we describe the properties and the information contained in the RASS-SDSS catalog and the resulting correlations between the X-ray and optical properties in the sample. In Sect. 2 we explain how the cluster sample has been created by X-ray selecting the systems from the available X-ray cluster and group catalogs. In Sect. 3 we describe the method for calculating the X-ray cluster properties. We describe in Sect. 4 the optical data and in Sect. 5 the data reduction method. We analyse and discuss the correlations between the optical luminosity and the X-ray properties in Sect. 6. We summarize and discuss the catalog properties and the results in Sect. 7.

For all derived quantities, we have used $H_{0}=$ $70 \mathrm{~km} \mathrm{~s}^{-1} \mathrm{Mpc}^{-1}, \Omega_{\mathrm{m}}=0.3$ and $\Omega_{\lambda}=0.7$.

\section{The construction of the sample}

In order to correlate optical and X-ray galaxy cluster properties it is necessary to have a large statistical sample and to cover the whole mass range of the systems considered. Since the $\mathrm{X}$-ray observations provide a robust method of identification of galaxy clusters and the X-ray luminosity is a good estimator of the system total mass, in principle the best approach should be to construct a cluster catalog of X-ray selected objects in a wide range of X-ray luminosity. While for intermediate and high X-ray luminosity (mass), several complete catalogs of X-ray selected clusters already exist (NORAS and REFLEX), in the low mass range a systematic X-ray survey of groups has still to be carried out. As a consequence, it is impossible at the moment to construct a strictly X-ray selected cluster sample, which covers a wide range of masses from very poor groups to rich clusters. A reasonable compromise in order to fill the low mass range of the spectrum is to select all the low mass 
clusters and groups X-ray detected to date, even if they are selected in other wavebands. This compromise is acceptable for our purposes, since we do not want to carry out an X-ray survey of galaxy clusters, but a systematic analysis of the correlation between X-ray and optical properties of those systems.

By following these criteria, the intermediate mass and high mass clusters have been selected from three ROSAT based cluster samples: the ROSAT-ESO flux limited X-ray cluster sample (REFLEX, Böhringer et al. 2002), the Northern ROSAT Allsky cluster sample (NORAS, Böhringer et al. 2000) and the NORAS 2 cluster sample (Retzlaff 2001). REFLEX is a complete sample of clusters detected in $13924 \mathrm{deg}^{2}$ in the southern hemisphere down to a flux limit of $3 \times 10^{-12} \mathrm{erg} \mathrm{s}^{-1} \mathrm{~cm}^{-2}$ in the $0.1-2.4 \mathrm{keV}$ energy bandcomprising 448 clusters. The NORAS galaxy cluster survey contains 495 clusters showing extended emission in the RASS in the northern hemisphere with count rates $C_{\mathrm{X}} \geq 0.06$ counts s${ }^{-1}$ in the $0.1-2.4 \mathrm{keV}$ energy band. NORAS 2 is the continuation of the NORAS project and aims at a complete survey of X-ray galaxy clusters, in $13598 \mathrm{deg}^{2}$ of the northern hemisphere, down to a flux limit of $2 \times 10^{-12} \mathrm{erg} \mathrm{s}^{-1} \mathrm{~cm}^{-2}$ in the same X-ray band, which gives rise to an expected total of about 800 clusters. The samples are based on an MPE internal X-ray source catalog extracted with a detection likelihood $\geq 7$.

The low mass clusters and the groups have been selected from two catalogs of X-ray detected objects: the ASCA Cluster Catalog (ACC) from Horner et al. (2001) and the Group Sample (GS) of Mulchaey et al. (2003). The ACC is a collection of all the clusters retrieved from the ASCA archive and discovered with different selection strategies. It contains measured luminosities, average temperatures, and metal abundances for 273 clusters and groups. The GS is a heterogeneous collection of 66 ROSAT systems, especially optically selected, with velocity dispersions less than $600 \mathrm{~km} \mathrm{~s}^{-1}$ or an intragroup medium temperature less than $2 \mathrm{keV}$.

The RASS-SDSS galaxy cluster sample comprises all the $\mathrm{X}$-ray clusters of the cited catalogs in the area covered by the Sloan Digital Sky Survey up to February 2003. For each X-ray system in the common RASS-SDSS area we found an optical counterpart. The sample includes 114 galaxy clusters, 14 of which come from REFLEX, 72 from NORAS and NORAS 2, 8 from Mulchaey's sample and 20 from ACC. The RASS-SDSS galaxy cluster sample, therefore, cannot be considered strictly an X-ray "selected" cluster sample, but should be defined more precisely an X-ray "detected" cluster sample because of the heterogeneous selection of the low mass range systems.

\section{The X-ray data}

To create a homogeneous catalog of X-ray cluster properties, we have calculated all X-ray parameters using only RASS data for all clusters in the sample. The X-ray luminosity has been calculated with the growth curve analysis (GCA) method used for REFLEX and NORAS 2, based on the RASS3 database (Voges et al. 1999). The GCA method was applied to RASS3 at the position of the clusters. The method allowed for a small adjustment of the position to center on the X-ray maximum. Since within the GCA aperture the measured flux is underestimated typically by an amount of 7-10\% (Böhringer et al. 2000) the missing flux is estimated by assuming an $\mathrm{X}$-ray luminosity scaled cluster model (Böhringer et al. 2001) and corrected for. A first approximate unabsorbed flux is calculated for each X-ray source from the observed count rate, by assuming a thermal spectrum with a temperature of $5 \mathrm{keV}$ and a metallicity of 0.3 solar and without a K-correction. Then an iterative computation uses the redshift and the unabsorbed $\mathrm{X}$-ray flux to give a first estimate of the luminosity. With the luminosity-temperature relation of Markevitch (1998, without correction for cooling flows) a better temperature estimate is obtained, and the count rate-flux conversion factor is recomputed including the appropriate K-correction for the redshift, resulting in a corrected flux and a new X-ray luminosity. The $\mathrm{X}$-ray luminosities as used in this paper are calculated in the ROSAT (0.1-2.4) keV energy band in the cluster rest frame for a $\Lambda$ cosmology with the parameters given above. The GCA also returns for each source many physical parameters like improved source position, background brightness, spectral hardness ratio, and KS probability for source extent.

The X-ray bolometric luminosity is derived from the $\mathrm{X}$-ray luminosity in the ROSAT $(0.1-2.4) \mathrm{keV}$ energy band. A first estimation of the cluster temperature is calculated using the $L_{\mathrm{X}}-T_{\mathrm{X}}$ relation of Xue \& $\mathrm{Wu}(2000)$ to estimate the appropriate bolometric correction.

For a subsample of 53 galaxy clusters we have also compiled from the literature the ASCA and XMM temperature and iron abundance of the intracluster medium in the system.

\section{Optical data}

The optical photometric data were taken from the SDSS (York et al. 2000; Stoughton et al. 2002). The SDSS consists of an imaging survey of $\pi$ steradians of the northern sky in the five passbands $u, g, r, i, z$, in the entire optical range from the atmospheric ultraviolet cutoff in the blue to the sensitivity limit of silicon in the red (Fig. 1). The survey is carried out using a $2.5 \mathrm{~m}$ telescope, an imaging mosaic camera with $30 \mathrm{CCDs}$, two fiber-fed spectrographs and a $0.5 \mathrm{~m}$ telescope for the photometric calibration. The imaging survey is taken in drift-scan mode. The imaging data are processed with a photometric pipeline (PHOTO) specially written for the SDSS data.

\subsection{The galaxy sample}

To study the optical cluster properties, we have created a complete galaxy sample for each cluster by selecting the galaxies in an area centered on the X-ray source position and within a radius of $1.5 \mathrm{deg}$. We used the selection criteria of Yasuda et al. (2001) to define our galaxy sample from the photometric catalog produced by PHOTO. We have selected only objects flagged with PRIMARY, to avoid multiple detections in the overlap between adjacent scan lines in two strips and between adjacent frames. Objects with multiple peaks (parent) are divided by the deblender into different components (children); if the objects can not be deblended, they are additionally flagged as NODEBLEND. Only isolated objects, child objects and NODEBLEND flagged objects are used in 


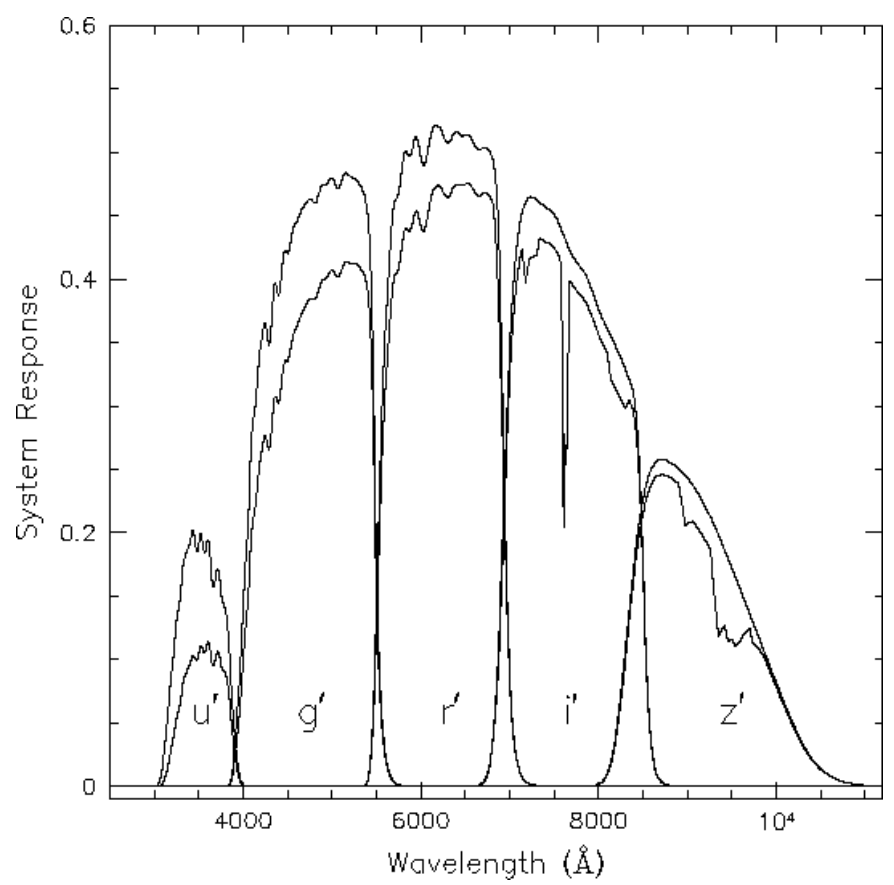

Fig. 1. Response function of the SDSS photometric system. Dashed curves indicate the response function including atmospheric transmission at 1.2 airmass at the altitude of Apache Point Observatory.

constructing our galaxy sample. The star-galaxy separation is performed in each band using an empirical technique based on the difference between the model and the PSF magnitude. An object is classified as galaxy if the model magnitude and the Point Spread Function (PSF) magnitude differ by more than 0.145 . This method seems to be robust for $r \leq 21$ mag, which is also the completeness limit of the survey in the Northern Galactic cap. Since saturated pixels and diffraction spikes can compromise the model-fitting algorithm, some stars can be misclassified as galaxies. Therefore we have rejected all object with saturated pixels and which are flagged as BRIGHT. Furthermore, we have classified an object as a galaxy only if PHOTO classified it as a galaxy in at least two of the three photometric bands $g, r, i$. After a visual inspection of a sample of galaxies with $r \leq 16$ mag, Yasuda et al. (2001) concluded that the described selection criteria give a sample completeness of $97 \%$, and the same completeness is found for the sample at $16 \leq r \leq 21$ mag after comparison with the Medium Deep Survey catalog (MDS) constructed using WFPC2 parallel images from HST. The major reason of missing real galaxies is the rejection of galaxies blended with saturated stars, while spurious galaxy detections are double stars or shredded galaxies with substructures.

\subsection{SDSS Galaxy photometry}

Since the galaxies do not have sharp edges or a unique surface brightness profile, it is nontrivial to define a flux for each object. PHOTO calculates a number of different magnitudes for each object: model magnitude, Petrosian magnitudes and PSF magnitudes. The model magnitudes are calculated by fitting de Vaucouleurs and exponential model, convolved with the local PSF, to the two dimensional images of the galaxies in the $r$ band. The total magnitude is determined from which of the two shape functions fits best. Galaxy colors are measured by applying the best fit model of an object in the $r$ band to the other bands, measuring the flux within the same effective aperture. However, due to a bug in PHOTO found during the completion of DR1, the model magnitudes are systematically underestimated by about 0.2 mag for galaxies brighter than 20th magnitude, and accordingly the measured radii are systematically too large (http://www.sdss.org/DR1/products/ catalogs/index.html). This error does not affect the galaxy colors but makes the model magnitudes useless for the determination of the galaxy total luminosities.

The Petrosian flux is defined by

$F_{\mathrm{p}}=2 \pi \int_{0}^{f_{2} r_{\mathrm{p}}} I(r) \mathrm{d} r$

where $I(r)$ is the surface brightness profile of the galaxy, and $r_{\mathrm{P}}$ is the Petrosian radius satisfying the equation:

$$
f_{1}=\frac{2 \pi \int_{0.8 r_{\mathrm{P}}}^{1.25 r_{\mathrm{P}}} \frac{I(r) r \mathrm{~d} r}{\pi\left[\left(1.25 r_{\mathrm{P}}\right)^{2}-\left(0.8 r_{\mathrm{P}}\right)^{2}\right]}}{2 \pi \int_{0}^{r_{\mathrm{P}}} \frac{I(r) r \mathrm{~d} r}{\left(\pi r_{\mathrm{p}}^{2}\right)}} .
$$

The Petrosian aperture is set to $2 r_{\mathrm{P}}$, and it encompasses $99 \%$ of the galaxy total light in case of an exponential profile and $82 \%$ in case of a de Vaucouleurs profile (Blanton et al. 2001). The Petrosian ratio $f_{1}$ is set to 0.2 ; at smaller values PHOTO fails to measure the Petrosian ratio, since the $S / N$ is too low. For faint objects the effect of the seeing on the Petrosian magnitude is not negligible. As the galaxy size becomes comparable to the seeing disk, the fraction of light measured by the Petrosian quantities approaches the fraction for a PSF, about 95\%, in which case the flux is reduced for a galaxy with an exponential profile and increased for a galaxy with a de Vaucouleurs profile (Strauss et al. 2002). Thus the Petrosian magnitudes are the best measure of the total light for bright galaxies, but fail to be a good measure for faint objects. In the data analysis of this paper we used the Petrosian magnitudes for galaxies brighter than 20 mag and the psf magnitudes for objects fainter than 20 mag.

\section{Optical luminosity from SDSS data}

\subsection{Background subtraction}

The total optical luminosity of a cluster has to be calculated after the subtraction of the foreground and background galaxy contamination. Since we have used only photometric data from the SDSS galaxy catalog, we have no direct information on the cluster galaxy memberships. There are two different approaches to overcome this problem. Since galaxy clusters show a very well defined red sequence in the color magnitude diagram, a galaxy color cut could be use to define the cluster membership (Gladders et al. 2000). On the other hand the background subtraction can be based on the number counts of the projected field galaxies outside the cluster. We chose the latter approach since the former method may introduce a bias against bluer cluster members. 


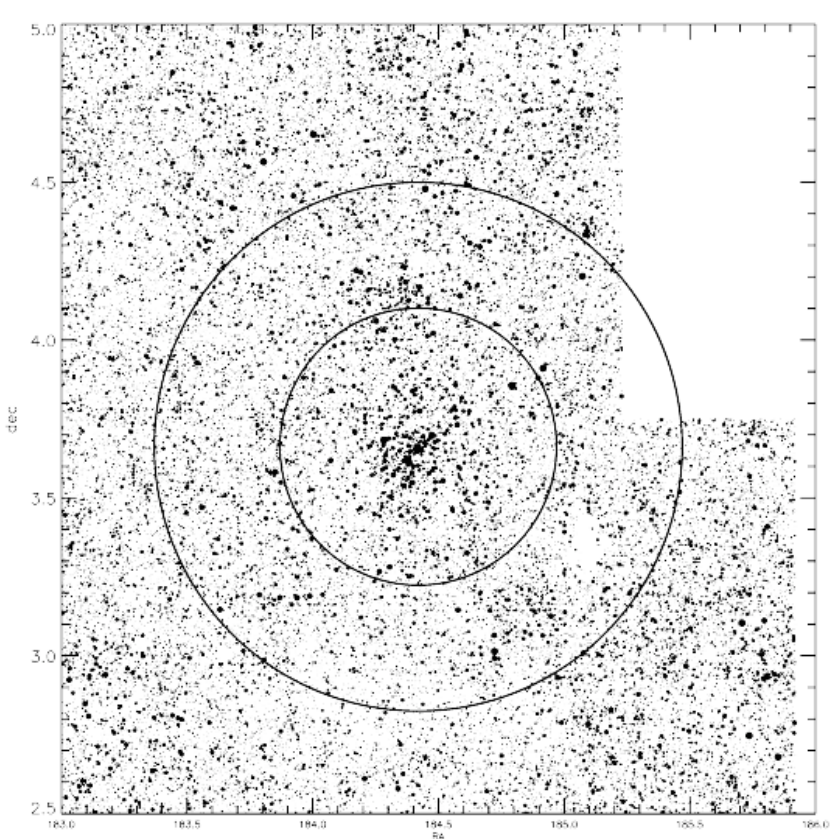

Fig. 2. The plot shows a cluster region with the local background. The dots represent the galaxies in the sample. The biggest dots correspond to the brightest galaxies in apparent $r$ magnitude. The local background number counts have been calculated inside the annulus with inner radius equal to $r_{200}+0.2 \mathrm{deg}$ and a width of 0.5 degree. The regions with voids due to lack of data or with close clusters have to be discarded in the background estimation.

We have considered two different approaches to the statistical subtraction of the galaxy background. First we have calculated a local background. The $M_{200}-L_{\mathrm{X}}$ relation of Reiprich \& Böringher (2002) was used to compute the $r_{200}$ radius (where the cluster mass density is 200 times the critical cosmic mass density), as a pragmatic approximation of the virial radius. Then, we defined an annulus centered on the cluster X-ray center, with an inner radius equal to $r_{200}+0.2 \mathrm{deg}$ and a width of 0.5 degree (Fig. 2). In this way the galaxy background has been estimated well outside the cluster but still locally. The annulus was then divided into 20 sectors (analogous to the approach in Böhringer et al. 2001) and those featuring a larger than $3 \sigma$ deviation from the median galaxy density were discarded from the further calculation. In this way other clusters close to the target or voids are not included in the background correction. We have computed the galaxies number counts $N_{\mathrm{bg}}^{l}(m) \mathrm{d} m$ per bin of magnitude (with a bin width of $0.5 \mathrm{mag}$ ) and per square degree in the remaining area of the annulus. The statistical source of error in this approach is the Poissonian uncertainty of the counts, given by $\sqrt{\left(N_{\mathrm{bg}}^{l}(m)\right)}$.

As a second method we have derived a global background correction. The galaxy number counts $N_{\mathrm{bg}}^{\mathrm{g}}(m) \mathrm{d} m$ were derived from the mean of the magnitude number counts determined in five different SDSS sky regions, each with an area of $30 \mathrm{deg}^{2}$ (Fig. 3). The source of uncertainty in this second case is systematic and originates in the presence of large-scale clustering within the galaxy sample, while the Poissonian error of the galaxy counts is small due to the large area involved. We have estimated this error as the standard deviation of the mean global

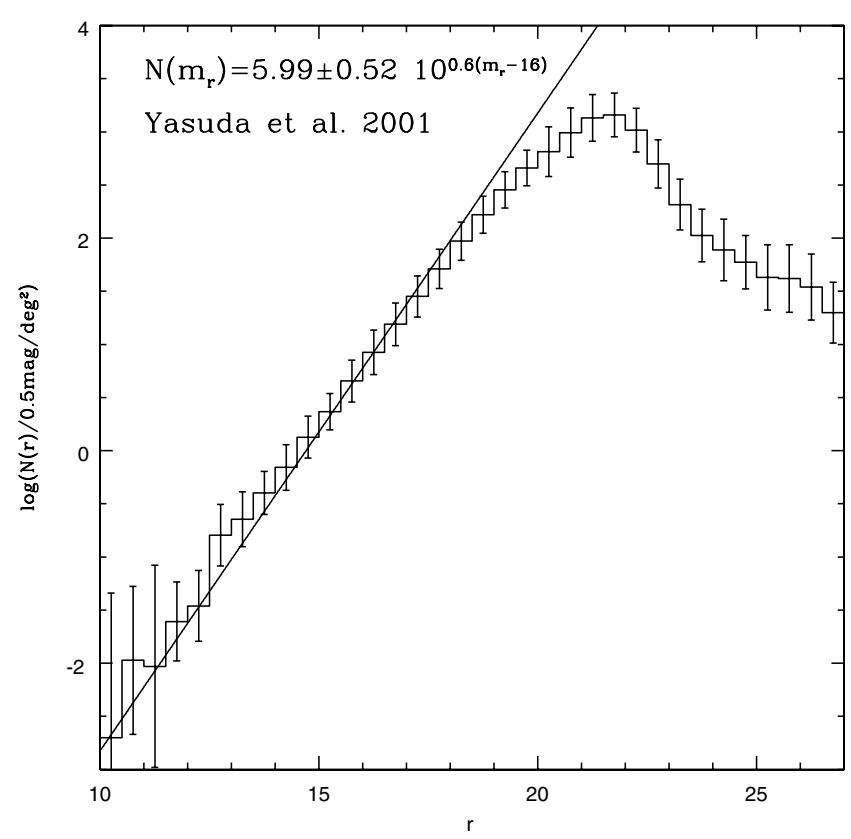

Fig. 3. Global background number counts as a function of magnitude in the $r$ band. The error bars include the contribution of large scale structure. The line shows the counts-magnitude relation expected for a homogeneous galaxy distribution in a universe with Euclidean geometry: $N(r)=A_{r} \times 10^{0.6(r-16)}$. The value of $A_{r}=$ $5.99 \pm 0.52(0.5 \mathrm{mag})^{-1} \mathrm{deg}^{-2}$ is the result of the fit in Yasuda et al. (2001) for $12 \leq r \leq 17$.

number counts, $\sigma_{\mathrm{bg}}^{\mathrm{g}}(m)$, in the comparison of the five areas. To take into account this systematic source of error also for the local background we have estimated the background number counts error as $\sigma_{\mathrm{bg}}(m)=\max \left(\sqrt{\left(N_{\mathrm{bg}}^{l}(m)\right)}, \sigma_{\mathrm{bg}}^{\mathrm{g}}(m)\right)$ (Lumdsen et al. 1997) for all the derived quantities.

After the background subtraction we found that the signal to noise in the $u$ band was too low to be useful, and performed our analysis on the 4 remaining Sloan photometric bands $g, r, i, z$.

\subsection{Luminosity function}

For each cluster and in all photometric bands we have assumed that the distribution function of galaxies in magnitude can be described by the Schechter Luminosity function (LF):

$$
\begin{aligned}
\phi(m) \mathrm{d} m= & 0.4 \ln (10) N_{\text {clu }} \phi^{*} 10^{-0.4\left(m-m^{*}\right)(\alpha-1)} \\
& \times \exp \left(-10^{-0.4\left(m-m^{*}\right)}\right) \mathrm{d} m
\end{aligned}
$$

In the equation $N_{\text {clu }}$ is the number of cluster galaxies and was computed as the difference between the total number of galaxies in the cluster region and the expected number of interlopers, estimated from the local (global) background galaxy density. $\phi^{*}$ is the normalization of the Schechter Luminosity function, given by the inverse of the integral of the LF over the considered magnitude range. To determine the remaining parameters $M^{*}$ and $\alpha$ we fitted the Schechter LF to the data with a Maximum Likelihood Method (MLM, Sarazin 1980). Since we have no information about the cluster membership, we have considered the observed galaxy magnitude distribution in the 
cluster region as the sum of the Schechter Luminosity function $(\phi(m))$ plus a background contribution $(b(m))$ :

$\Phi(m)=\frac{\phi(m)+b(m)}{N_{\text {tot }}}$

$\Phi(m)$ is normalized to unity when integrated over the considered range of magnitudes.

To perform an ML analysis, the background contribution has to be specified at any magnitude. To estimate the $b(m)$, one can try to fit the background number counts by a model. While the behavior of the $N(m)-m$ relation is well know at the bright end (in the $\log N(m)-\log m$ it is a line with slope 0.6, Fig. 3), it is not well understood at the faint end (Yasuda et al. 2001; Lumdsen et al. 1997). Therefore instead of using a specific functional form, we simply have used a spline to interpolate the background galaxy number counts and estimated $b(m)$ at any magnitude.

The probability that the assumed distribution gives a galaxy at the magnitudes $m_{k}$ is thus $\Phi\left(m_{k}\right)$. Therefore, if the observed galaxies are statistically independent, the combined probability that the assumed distribution gives the observed galaxies at the magnitude $m_{k}$ (with $k=1, n$ ) is:

$L=\prod_{\kappa=1}^{n} \Phi\left(m_{k}\right)$

The best-fit parameters are those that maximize the likelihood $L$. In practice we have minimized the log-likelihood $-2 \ln (L)$. This minimization was performed with the CERN software package MINUIT. We used the variable metric method MIGRAD (Fletchter 1970) for the convergence at the minimum, and the MINOS routine to estimate the error parameters in case of non-linearities. We also have placed constraints on the values of $m^{*}$ and $\alpha$ that the fitting routine can accept, to avoid being trapped in a false minimum $(M *$ in the range between -18 and -26 mag and $\alpha$ between 0 and -2.5 , Lumdsen et al. 1997). Figure 7 gives an example of the LF derived with the MLM in each of the Sloan photometric bands for a cluster. Figures 4 and 5 show the comparison between the fit parameters calculated with different backgrounds $b(m)$ (local and global). Figure 6 shows the correlation between the fit parameters $M^{*}$ and $\alpha$.

The great advantage of the MLM is that the method does not require the data to be binned and does not depend on the bin size, but uses all the available information. On the other hand, the MLM provides no information about the goodness of the fit. Therefore, we have performed a statistical test. Since the routine procedure uses a unbinned set of data to perform the fit, in principle the Kolmogorov Smirnov test should be applicable to our case. Nevertheless since the KS probability is not easy to interpret, we have applied a $\chi^{2}$ test, by comparing the background subtracted magnitude number counts of the cluster with the Schechter luminosity function fitted to the data. Figure 8 shows the distribution of the reduced $\chi^{2}$ in the cluster sample. Almost $90 \%$ of the fitted LFs are a good fit to the data having a reduced $\chi^{2} \leq 1$.

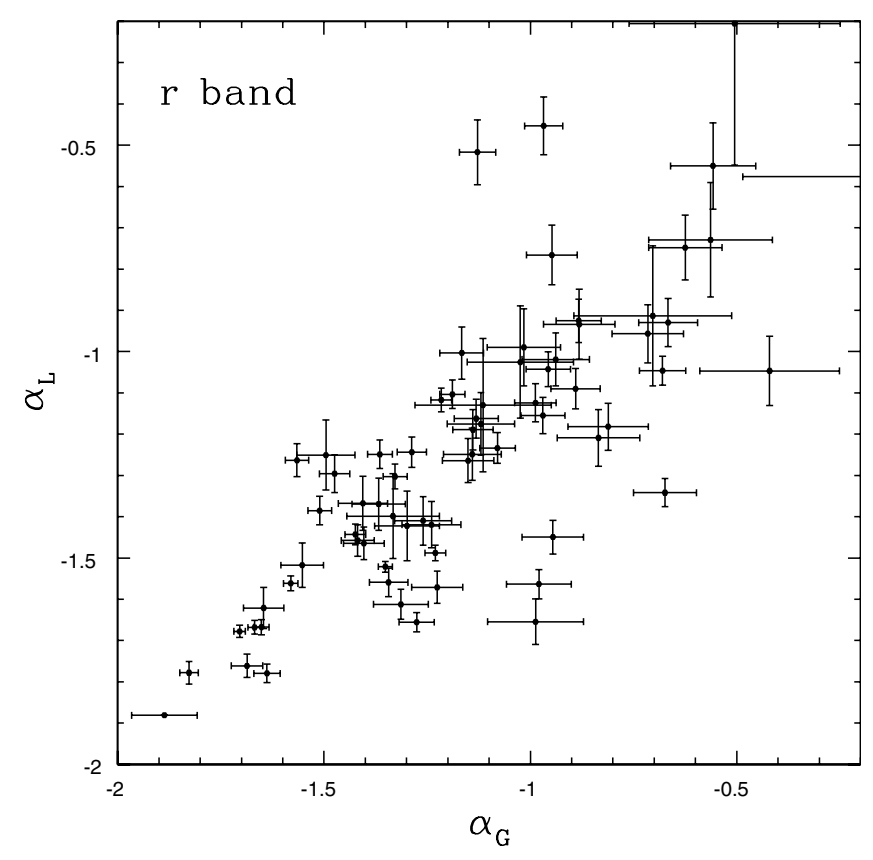

Fig. 4. Comparison of the $\alpha$ parameter (slope) of the individual Schechter luminosity functions. $\alpha_{\mathrm{L}}$ is calculated using the local background correction, while $\alpha_{\mathrm{G}}$ is the result of the global background correction. The different background subtractions give consistent results. The error bars in the plot are at the $68 \%$ confidence level.

\subsection{The total luminosity}

To calculate the total cluster luminosity we have calculated first the absolute magnitude

$M=m-25-5 \log _{10}\left(D_{\mathrm{L}} / 1 \mathrm{Mpc}\right)-A-\mathrm{K}(z)$

where $D_{\mathrm{L}}$ is the luminosity distance, A is the Galactic extinction and $\mathrm{K}(z)$ is the $\mathrm{K}$-correction. We deredden the Petrosian and model magnitudes of galaxies using the Galactic map of Schlegel et al. (1998) in each photometric band. We used the K-correction supplied by Fukugita et al. (1995) for elliptical galaxies, assuming that the main population of our clusters are the old elliptical galaxies at the cluster redshift. The transformation from absolute magnitudes to absolute luminosity in units of solar luminosities is performed using the solar absolute magnitude obtained from the color transformation equation from the Johnson-Morgan-Cousins system to the SDSS system of Fukugita et al. (1996). We have calculated the optical luminosity of each cluster with two different methods. First, we have estimated $L$ by using the (background corrected) magnitude number counts of the cluster galaxies with the following prescription:

$L=\sum_{i}^{N} N_{i}(m) l_{i}(m)+\int_{m_{\lim }}^{\infty} \phi(m) \mathrm{d} m$.

The sum on the right side is performed over all the $N$ magnitude bins with galaxy number $N_{i}(m)$ and mean luminosity $l_{i}(m)$. The integral is an incompleteness correction due to the completeness limit of the galaxy sample at $m_{\mathrm{lim}}=21 \mathrm{mag}$ in the five Sloan photometric bands. $\phi(m)$ is the individual Schechter 


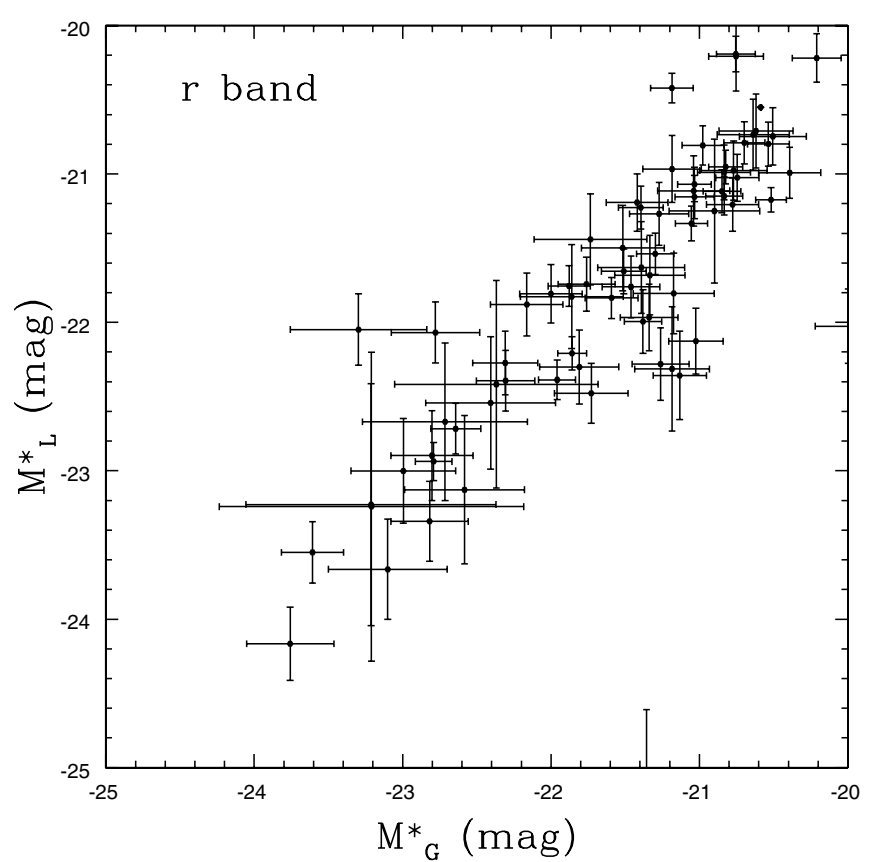

Fig. 5. Comparison of the $M *$ parameter (knee) of the individual Schechter luminosity functions. $M *_{\mathrm{L}}$ is calculated using the local background correction, while $M *_{\mathrm{G}}$ is the result of the global background correction. The different background subtractions give consistent results, as they do for the slope. The error bars in the plot are at $68 \%$ confidence level.

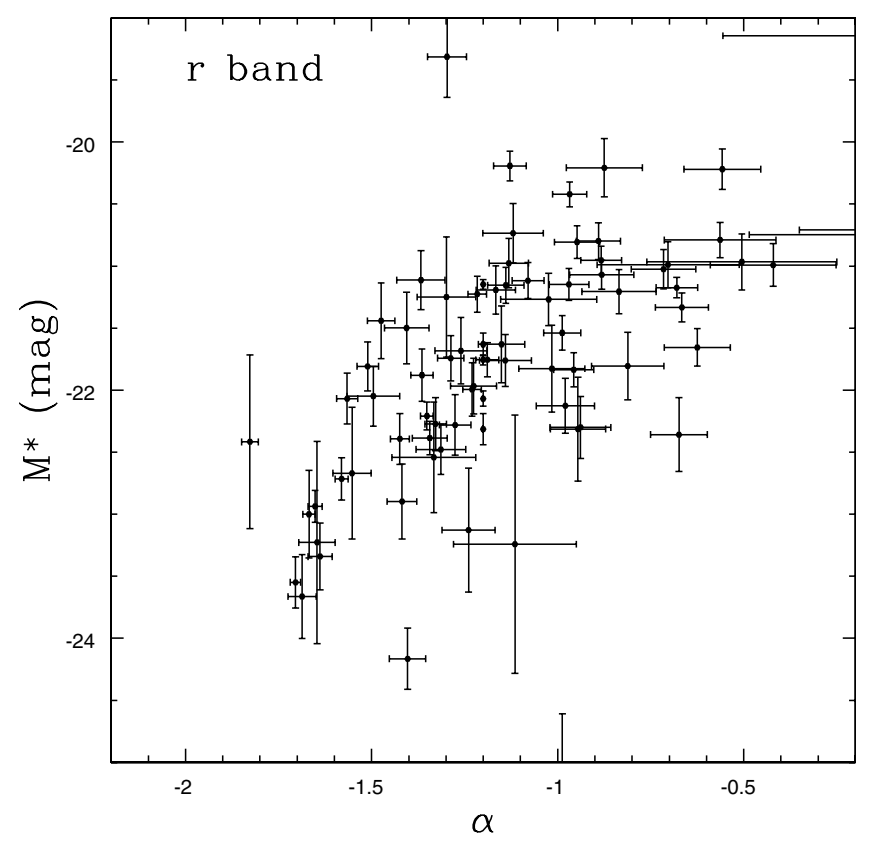

Fig. 6. Fit parameters of the individual Schechter luminosity functions in the $r$ band. The fitting procedure is performed using the local background magnitude number counts. The parameters show a slight correlation. The error bars in the plot are at the $68 \%$ confidence level.

luminosity function fitted to the galaxy sample of each cluster. The incompleteness correction is of the order of $5-10 \%$ in the whole cluster sample, as shown in Fig. 9. This means that the galaxies below the magnitude limit do not give a significant contribution to the total optical luminosity. Therefore the
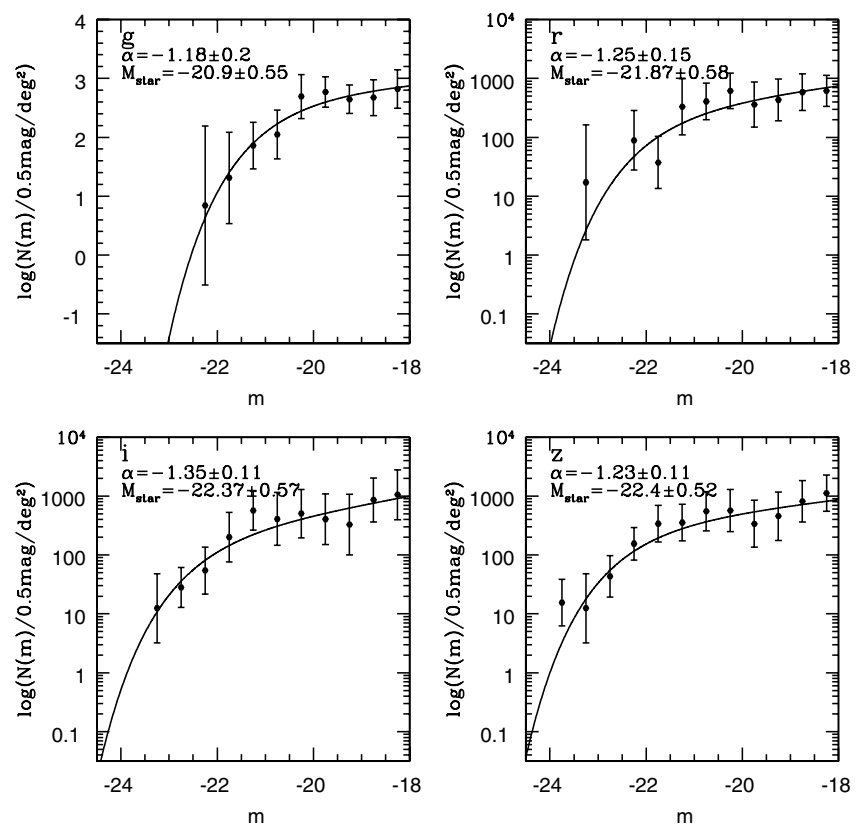

Fig. 7. Example of the individual fitted luminosity function of a cluster in 4 Sloan photometric bands. The values of the fitted parameters are indicated in each panel.

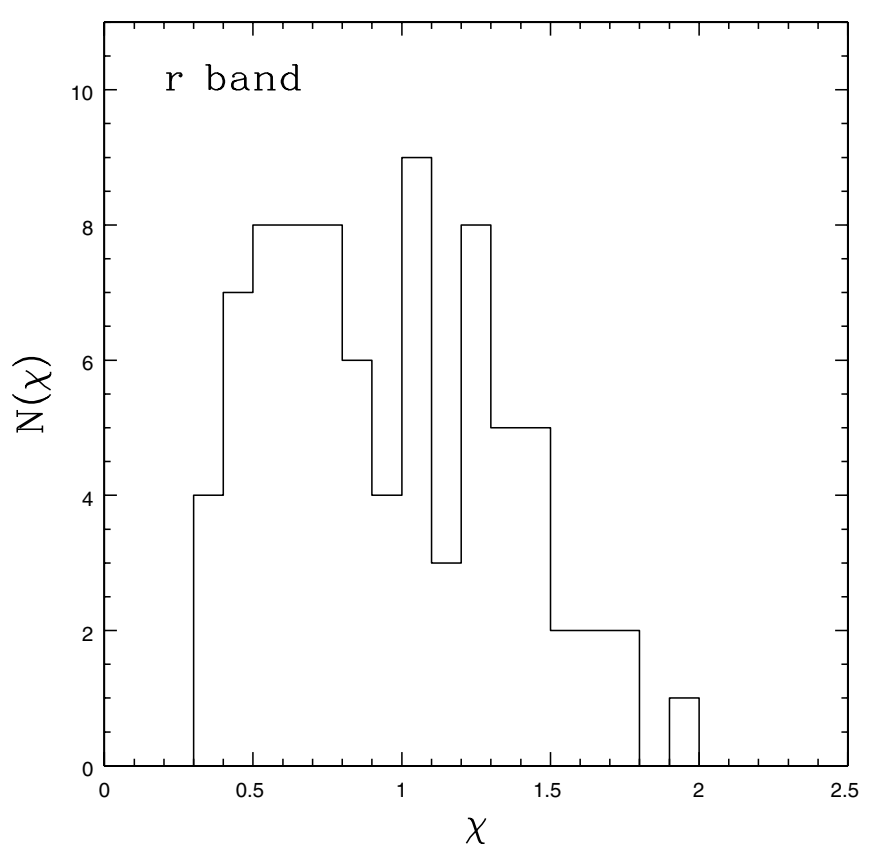

Fig. 8. Distribution of the reduced $\chi^{2}$ of the individual Schechter luminosity functions. Since the MLM allows us to perform a fit without binning the data but does not give information about the goodness of the fit, we have performed the fitting procedure with MLM and checked the goodness of our fitted luminosity functions with a $\chi^{2}$ test. All clusters are well represented by the individual fitted luminosity functions.

most important source of error is due to the contribution of the background galaxy number counts. The uncertainty in each bin of magnitude is given by the Poissonian error of the bin counts $\left(\sqrt{N_{\mathrm{tot}}^{i}(m)}\right.$, with $\left.i=1, \ldots, N\right)$ and the background subtraction in each magnitude bin $\left(\sigma_{\mathrm{bg}}^{i}(m)\right.$, with $i=1, \ldots, N$, see previous 


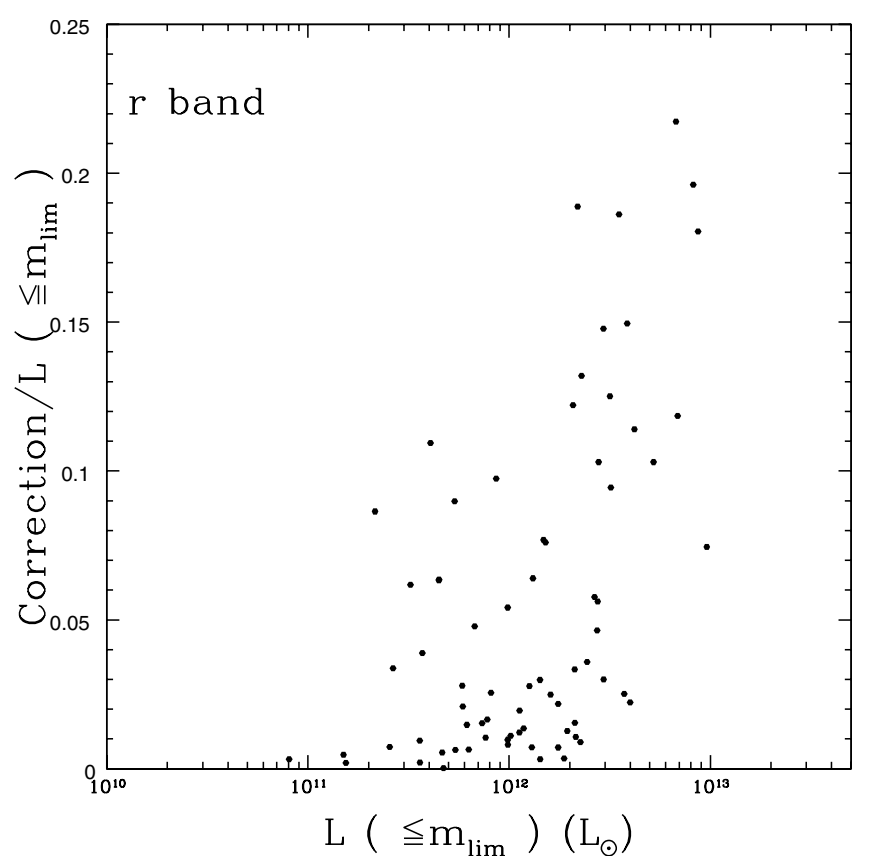

Fig. 9. The plot shows the correction to the total optical luminosity for incompleteness. The completeness magnitude limit is 21 mag in each Sloan photometric band. The correction is of the order of 5\% for $50 \%$ of the clusters in the sample, while it is less than $10 \%$ for $85 \%$ of the sample. The trend in the plot is due to a selection effect, since the most distant clusters are also the most luminous ones.

section for the definition). Since the galaxy counts in the bins are independent, the error in the luminosity is given by:

$\Delta L=\left(\sum_{i}^{N}\left(N_{\mathrm{tot}}^{i}(m)+\sigma_{\mathrm{bg}}^{i}(m)^{2}\right)\right)^{\frac{1}{2}}$.

Figure 10 shows the comparison between the luminosity calculated from the local background corrected and global background corrected magnitude number counts. The difference between the two methods is much smaller than the statistical error.

In the second case we have taken advantage of the individual fitted luminosity function. If MIGRAD has converged successfully to the minimum, we calculate the total luminosity as

$L=L^{*} N_{\mathrm{clu}} \phi^{*} \Gamma(2+\alpha)$

where $L^{*}$ and $\alpha$ are the fit parameters estimated from the data and $N_{\text {clu }}$ is the total number of cluster galaxies. There are different sources of errors in this calculation. The major source of errors comes from the background which affects both the number of cluster galaxies $N_{\text {clu }}$ and the result of the fitting procedure. A second kind of error is due to the uncertainty of the fit parameters. Since all these errors are not independent, we can not treat their contributions separately. Therefore the luminosity errors were calculated by varying the fit parameter values, $M^{*}$ and $\alpha$, along their $68 \%$ confidence level error ellipse and using the upper and lower bound of the quoted background number counts $(b(m))$ range. The statistical luminosity error was then defined as half of the difference between the minimum and maximum

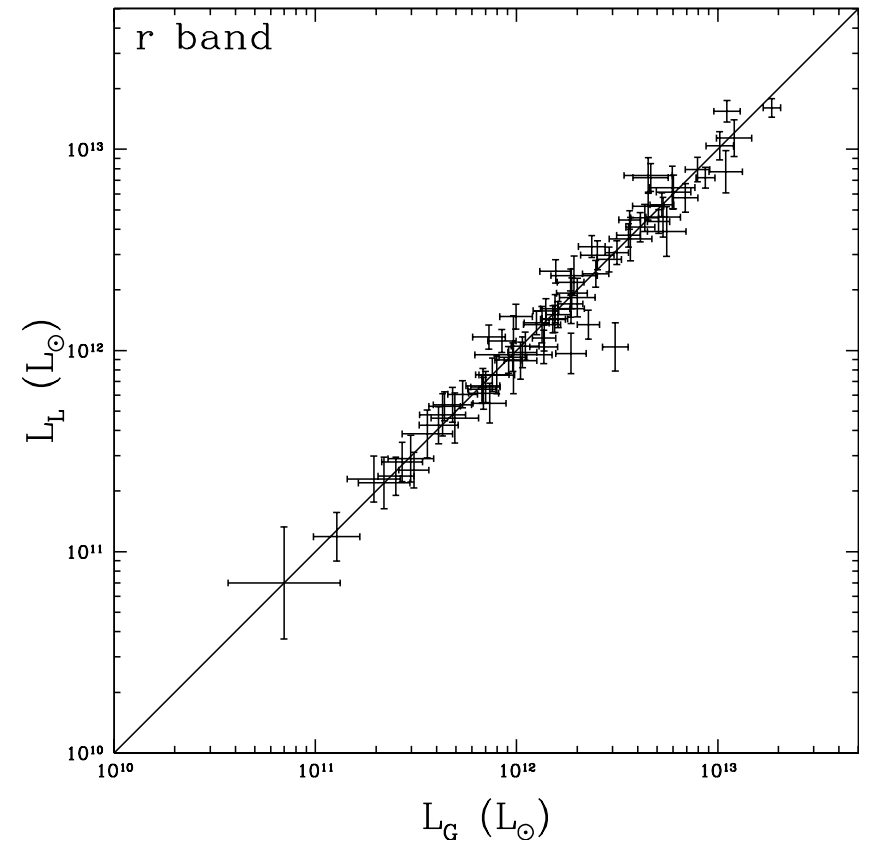

Fig. 10. Comparison of the cluster optical luminosities calculated from the cluster magnitude number counts with different background corrections. $L_{\mathrm{L}}$ is calculated with the local, and $L_{G}$ with the global background subtraction. The different corrections do not affect the cluster luminosity estimation. The error bars in the plot are at the $68 \%$ confidence level.

luminosity. With this method we can take into account statistical and systematic errors due to the background, and their effects on the fit parameters as well. Note that a simple error propagation applied to Eq. (9) would underestimate the error in the luminosity, since it would not take into account the error of the galaxy background.

Figure 11 shows the comparison of the two optical luminosities (fit-based and count-based), which are consistent within the errors. For $70 \%$ of the clusters in the sample the luminosity based on counts is systematically brighter than that based based on fit, as shown in Fig. 12. In the former case, indeed, the method includes in the calculation of $L_{\mathrm{op}}$ the Bright Cluster Galaxies (BCG) which are usually excluded by the Schechter luminosity function. The error bars in the fit-based luminosity are larger than in the count-based luminosity. In fact in the former case there are two main sources of error: the uncertainty due to the galaxy background subtraction and the statistical errors in the fit parameters of the luminosity function. In the latter case, instead, only the subtraction of the galactic background plays a crucial role. The mean error in the fitbased luminosity is around 30\%, while it is around $20 \%$ with the count-based method.

The great advantage of the count-based optical luminosity is that it can be easily computed, if the cluster $S / N$ is high enough. On the other hand, the fit-based luminosity depends on the success of the fitting procedure. Therefore, it is sensitive not only to the $S / N$ but also to the chosen model and the goodness of the fit. The uncertainty in the count-based method is smaller than in the fit-based method. Moreover, while the count-based method provides the optical luminosity for any system and at 


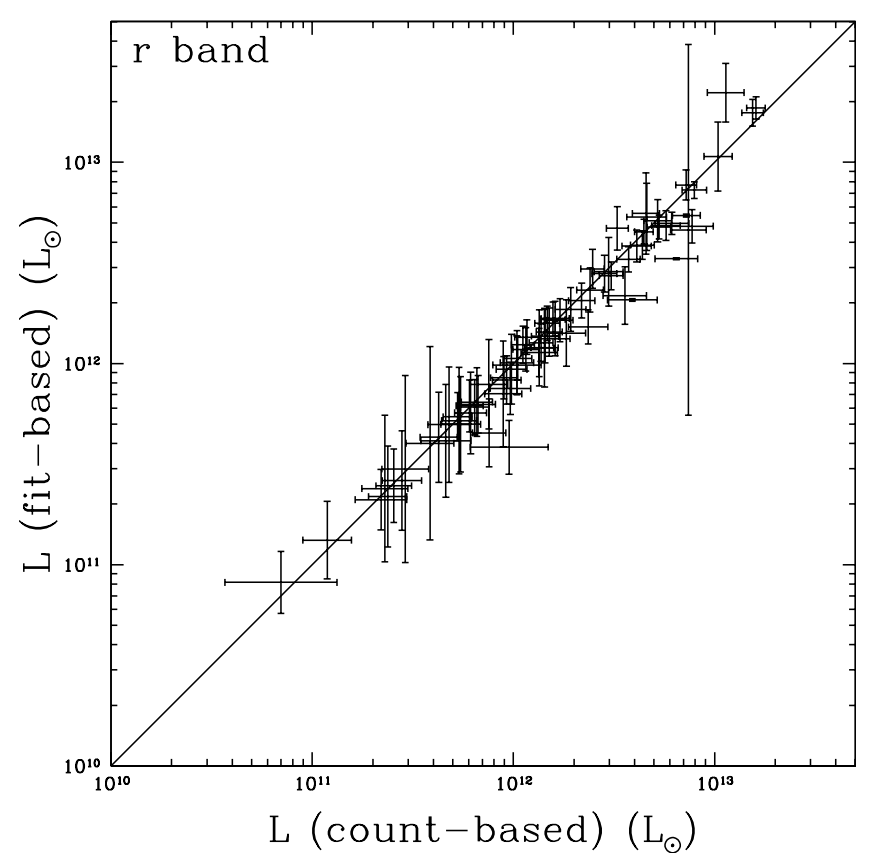

Fig. 11. Comparison of the cluster luminosities determined with count-based and fit-based methods. The error bars in the plot are at the $68 \%$ confidence level.

any cluster aperture, the number of failures in the fitting procedure is an increasing function of the cluster aperture. In fact the fit-based method fails to fit the data for $15 \%$ of the clusters at $0.35 \mathrm{Mpc} h_{70}^{-1}$ and up to $35 \%$ at $2.0 \mathrm{Mpc} h_{70}^{-1}$. In consequence, the count-based $L_{\mathrm{op}}$ has to be preferred to the fit-based one in the study of the correlation between optical and X-ray properties. The count-based method also reflects what we actually observe.

On the basis of this analysis we can conclude that the behavior of the optical luminosities calculated with different background subtraction is stable for variant approaches and the main source of errors is due to the necessary background subtraction (Fig. 10). Moreover, since the two different methods (count-based and fit-based) give consistent results, our measure of $L_{\mathrm{op}}$ seems to be a good estimation of the cluster total optical luminosity.

\subsection{The optical structure parameters}

To study the spatial distribution of galaxies in cluster we have analysed the projected radial galaxy distributions of all clusters in the sample. The analysis is performed in the $g, r, i$ and $z$ bands. As for the luminosity functions, we have used a Maximum Likelihood method to fit a King profile to the data

$P(r)=\frac{\sigma_{0}}{\left(1+\left(\frac{r}{r_{\mathrm{c}}}\right)^{2}\right)^{\beta}}+\sigma_{\mathrm{b}}$.

In Eq. (10), $\sigma_{0}$ is the central galaxy density, $r_{\mathrm{c}}$ the core radius, $\beta$ the profile exponent, and $\sigma_{\mathrm{b}}$ the background density. $P(r)$ has to be normalized through:

$\int_{A} P(r) \mathrm{d}\left(\pi r^{2}\right)=N_{\text {tot }}$

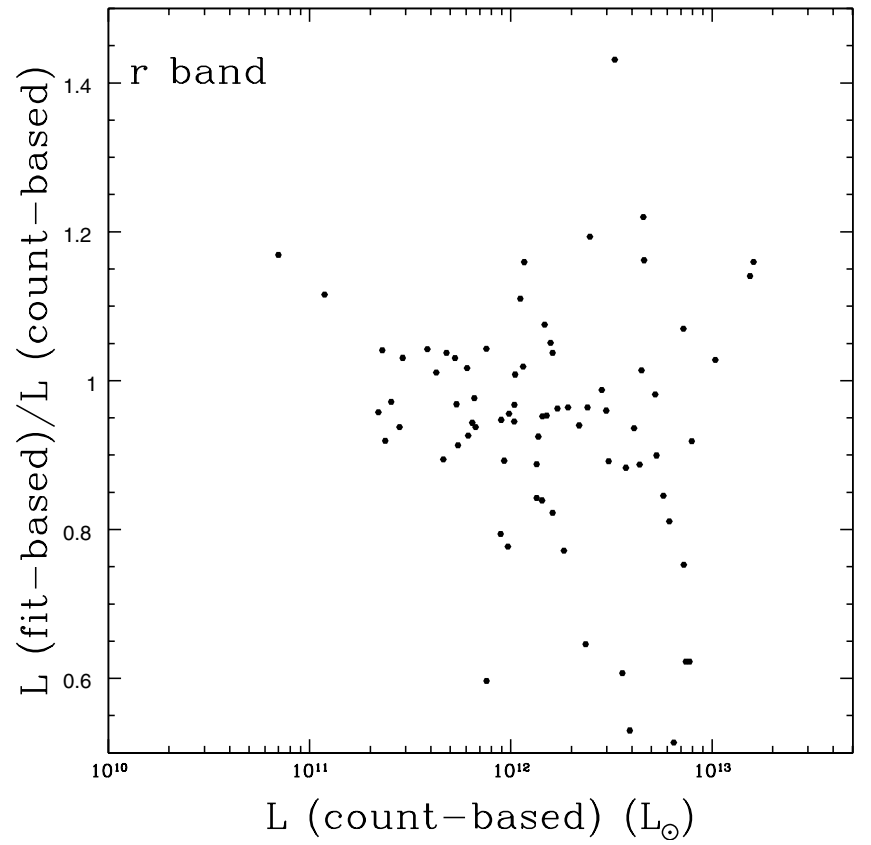

Fig. 12. Comparison of the count-based and fit-based cluster luminosities. The plot shows the ratio of these two versus the count-based luminosity. For $70 \%$ of the sample the ratio $L_{\text {fit-based }} / L_{\text {count-based }}$ is less than 1.1, indicating that the fit-based luminosity is systematically less bright the count-based luminosity. This is due to the Bright Cluster Galaxies (BCG): they are included in the computation of the countbased luminosity but not in the estimation of the fit-based luminosity.

where $A$ is the relevant cluster area and $N_{\text {tot }}$ is the total number of galaxies within that area. In agreement with Sect. 5.2 the Likelihood is given by:

$L=\prod_{\kappa=1}^{n} P\left(r_{\kappa}\right)$

where $r_{k}$ is the projected galaxy distance from the X-ray center. We regarded $\beta, r_{\mathrm{c}}$ and $\sigma_{\mathrm{b}}$ as fitting parameters, while $\sigma_{0}$ is a dependent variable and its value is derived from the likelihood normalization, Eq. (11). The fitting method worked successfully on average for $95 \%$ of the sample in any photometric band; it failed for groups, where the overdensity in comparison to the background density is too low to fit a profile. As shown in Fig. 13, there are no correlations of the parameters $\sigma_{0}, r_{\mathrm{c}}$, and $\beta$ with the background density $\sigma_{\mathrm{b}}$. Furthermore the histogram of the $\beta$ values in the same figure shows that the mean value of the profile exponent is around 0.8 with a very large dispersion of 0.5 around the peak.

We have estimated from the King profile the physical size of each cluster, $r_{\text {tot }}$. We have assumed that this quantity is given by the radial distance from the X-ray center, where the galaxy number density of the cluster becomes $n$ times the error of the background galaxy density (the cross in Fig. 14). To search for the best value of $n$, we have estimated the total radius with different values of $n(n=1, \ldots, 5)$ and calculated the total optical luminosity within that radius; $n$ was then fixed to 3 , since the differences in the luminosities calculated within different total radii are smaller than the luminosity uncertainties due to background subtraction. 

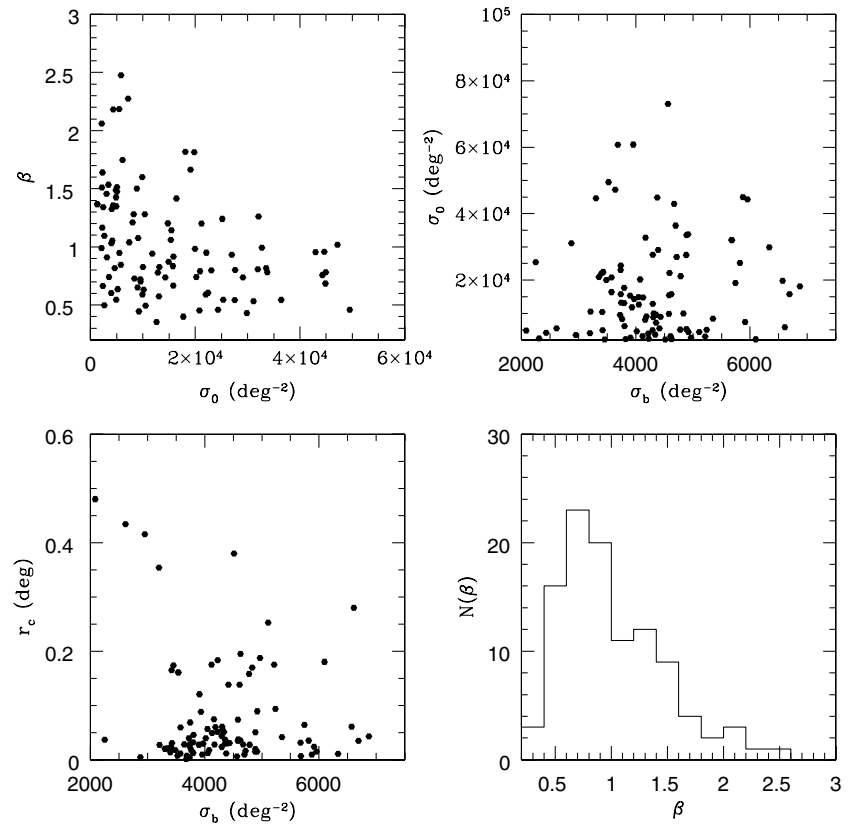

Fig. 13. The four panels show the behavior of the fit parameters in the King profile fit procedure. The first three panels show that there is no correlation between the parameters, while the bottom right panel presents the histogram of the exponent $\beta$ of the King profile.

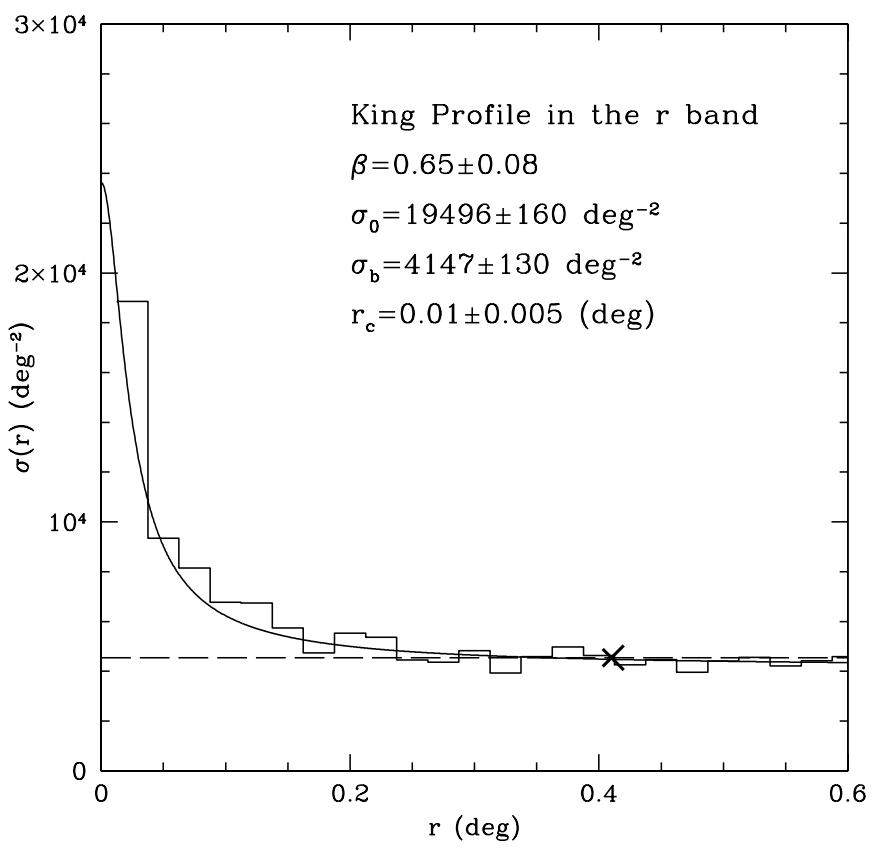

Fig. 14. The physical total size (the cross) of each cluster is estimated as the radius where the galaxy number density within the cluster becomes 3 times the error in the statistical background galaxy number density (the dashed line).

We assumed that the cluster total optical luminosity in a band is the luminosity calculated within $r_{\text {tot }}$ estimated in the given filter. To calculate then the half-light radius, which encircles half of the total cluster luminosity, we estimated in each band the luminosity of the cluster within 20 increasing apertures centered in the X-ray center. We obtained the cluster cumulative luminosity profile interpolating in each filter the
20 values of the luminosity calculated within increasing apertures. Finally we used the cluster cumulative luminosity profile to find the radius which corresponds to half of the total cluster luminosity.

\subsection{The catalog}

In the following we present the catalog of the 114 RASS-SDSS galaxy clusters. Tables 1-3 list all the X-ray and optical properties of the sample computed as explained in the previous sections. The sample tables show the results for the first 35 clusters in the sample. The complete tables are available in electronic form.

Table 1 gives the $\mathrm{X}$-ray properties of the cluster derived from the ROSAT data. Columns 1 and 2 put the ROSAT and the alternative cluster name respectively. Columns 3 and 4 contain the equatorial coordinates of the X-ray cluster center used for the region selection for the epoch J2000 in decimal degrees. Column 5 contains the heliocentric cluster redshift. Column 6 presents the flux in the energy band $0.1-2.4 \mathrm{keV}$ in units of $10^{-11} \mathrm{erg} \mathrm{s}^{-1} \mathrm{~cm}^{-2}$. Column 7 gives the corrected flux for a temperature derived from the $L_{X}-T$ relation including the K-correction for an assumed cluster temperature of $5 \mathrm{keV}$. Column 8 contains the relative $1 \sigma$ Poissonian error of the count rate, the flux and the luminosity in percents. Column 9 gives the luminosity in the energy range $0.1-2.4 \mathrm{keV}$ in units of $h_{70}^{-2} \times 10^{44} \mathrm{erg} \mathrm{s}^{-1}$. Column 10 contains the count rate in units of counts $\mathrm{s}^{-1}$. Column 11 gives the outer radius within which the flux and the luminosity are estimated, in units of arc minutes.

Table 2 provides the optical parameters of the luminosity function and the luminosities of each cluster calculated using the local galaxy background. Listed are results in the $r$ band. All the quantities are calculated within a fixed cluster aperture of $1.0 \mathrm{Mpc} h_{70}^{-1}$. Column 1 presents the ROSAT name of the cluster. Columns 2 and 3 show the resulting fit parameters of a Schechter luminosity function. $\alpha$ is the slope of the LF, while $M *$ is the magnitude knee of the distribution. Column 4 gives the galaxy number density within the cluster region selected to perform the fit, in units of $\mathrm{deg}^{-2}$. Column 5 shows the reduced $\chi^{2}$ of the fitted luminosity function. Column 6 provides the cluster optical luminosity in units of $10^{12} L_{\odot}$, calculated on the basis of the fitted luminosity function. Column 7 lists the cluster optical luminosity in units of $10^{12} L_{\odot}$, calculated on the basis of the cluster magnitude number counts. All the errors in the table are at $68 \%$ confidence level. The full catalog, which is an extended version of this table, is available online. Similar tables exist, listing the parameters and the luminosities relative to 22 different cluster apertures: 20 fixed apertures ranging from 0.05 to $4.0 \mathrm{Mpc} h_{70}^{-1}, 2$ variable apertures as the core radius and the half-light radius. All the data are provided in each of the 4 Sloan photometric bands $g, r, i$ and $z$, and for both local and global galaxy background correction.

Table 3 provides the information concerning the radial distribution of the projected galaxy density in the region of the cluster. Column 1 presents the ROSAT name of the cluster. Column 2 gives the cluster central galaxy number density in 
Table 1. Example of the table containing the X-ray cluster properties of the whole cluster sample. The table show the results for the first 35 cluster in the sample.

\begin{tabular}{|c|c|c|c|c|c|c|c|c|c|c|}
\hline \multicolumn{3}{|c|}{ Alternative } & \multirow[b]{2}{*}{$\begin{array}{l}\text { Dec } \\
(4)\end{array}$} & \multirow[b]{2}{*}{$\begin{array}{c}z \\
(5)\end{array}$} & \multirow[b]{2}{*}{$\begin{array}{l}F_{X} \\
(6)\end{array}$} & \multirow[b]{2}{*}{$\begin{array}{l}F_{\mathrm{X}}{ }^{*} \\
(7)\end{array}$} & \multirow[b]{2}{*}{$\begin{array}{l}\text { Error } \\
(8)\end{array}$} & \multicolumn{3}{|c|}{$\overline{\text { Count }}$} \\
\hline $\begin{array}{l}\text { Name } \\
(1)\end{array}$ & $\begin{array}{c}\text { Name } \\
\text { (2) }\end{array}$ & $\begin{array}{l}\text { RA } \\
\text { (3) }\end{array}$ & & & & & & $\begin{array}{l}L_{\mathrm{X}} \\
(9)\end{array}$ & $\begin{array}{l}\text { rate } \\
(10)\end{array}$ & $\begin{array}{l}R_{\text {out }} \\
(11)\end{array}$ \\
\hline RXC J0041.8-0918 & $A 0085$ & 10.4587 & -9.3019 & 0.052 & 67.612 & 67.905 & 3.0 & 7.87 & 3.25 & 18.0 \\
\hline RXC J 0114.9+0022 & $A 0168$ & 18.7350 & 0.3746 & 0.047 & 8.725 & 8.484 & 8.7 & 0.81 & 0.42 & 14.0 \\
\hline RXC J0119.6+1453 & $A 0175$ & 19.9072 & 14.8931 & 0.129 & 3.124 & 3.114 & 29.1 & 2.23 & 0.14 & 9.5 \\
\hline RXC J0137.2-0912 & $\ldots$ & 24.3140 & -9.2028 & 0.039 & 7.275 & 7.071 & 8.4 & 0.46 & 0.35 & 9.5 \\
\hline RXC J0152.7+0100 & $A 0267$ & 28.1762 & 1.0126 & 0.227 & 4.257 & 4.276 & 12.1 & 9.32 & 0.20 & 9.0 \\
\hline RXC J0736.4+3925 & $\ldots$ & 114.1040 & 39.4329 & 0.117 & 8.239 & 8.239 & 9.5 & 4.81 & 0.36 & 13.0 \\
\hline RXC J0747.0+4131 & $\ldots$ & 116.7537 & 41.5314 & 0.028 & 3.201 & 2.431 & 15.0 & 0.08 & 0.14 & 9.5 \\
\hline RXC J0753.3+2922 & $\ldots$ & 118.3291 & 29.3741 & 0.062 & 6.414 & 0.062 & 9.6 & 1.04 & 0.30 & 9.0 \\
\hline RXC J0758.4+3747 & $N g c 2484$ & 119.6172 & 37.7888 & 0.041 & 0.605 & 0.431 & 32.1 & 0.03 & 0.02 & 7.0 \\
\hline RXC J0800.9+3602 & $A 0611$ & 120.2445 & 36.0469 & 0.288 & 2.536 & 2.545 & 16.9 & 8.85 & 0.11 & 6.0 \\
\hline RXC J0809.6+3455 & $\ldots$ & 122.4177 & 34.9262 & 0.080 & 5.208 & 5.164 & 13.2 & 1.43 & 0.24 & 7.5 \\
\hline RXC J0810.3+4216 & $\ldots$ & 122.5942 & 42.2669 & 0.064 & 2.974 & 2.893 & 13.8 & 0.51 & 0.13 & 6.0 \\
\hline RXC J0822.1+4705 & $A 0646$ & 125.5417 & 47.0995 & 0.130 & 7.236 & 7.236 & 9.0 & 5.25 & 0.34 & 8.0 \\
\hline RXC J0824.0+0326 & $M S 0821.5+0337$ & 126.0209 & 3.4383 & 0.347 & 0.297 & 0.294 & 78.6 & 1.57 & 0.01 & 2.5 \\
\hline RXC J0825.4+4707 & $A 0655$ & 126.3652 & 47.1196 & 0.126 & 7.235 & & 15.1 & 4.92 & 0.34 & 12.0 \\
\hline RXC J0828.1+4445 & $\ldots$ & 127.0278 & 44.7634 & 0.145 & 4.501 & 4.501 & 11.2 & 4.04 & 0.21 & 6.0 \\
\hline RXC J0842.9+3621 & $A 0697$ & 130.7401 & 36.3625 & 0.282 & 5.821 & 5.858 & 16.0 & 19.42 & 0.28 & 8.0 \\
\hline RXC J0845.3+4430 & $H G C 35$ & 131.3434 & 44.5115 & 0.054 & 0.082 & 0.057 & 100.0 & 0.00 & 0.00 & 0.5 \\
\hline RXC J0850.1+3603 & $C L 0847.2+3617$ & 132.5499 & 36.0614 & 0.373 & 2.742 & 2.75 & 18.7 & 15.87 & 0.13 & 9.5 \\
\hline RXC J0913.7+4056 & CL09104 + 4109 & 138.4411 & 40.9339 & 0.442 & 1.756 & 1.769 & 30.1 & 14.16 & 0.09 & 8.5 \\
\hline RXC J0913.7+4742 & $A 0757$ & 138.4446 & 47.7021 & 0.051 & 6.202 & 6.022 & 13.3 & 0.68 & 0.31 & 15.0 \\
\hline RXC J0917.8+5143 & $A 0773$ & 139.4637 & 51.7223 & 0.217 & 5.961 & 5.998 & 9.2 & 11.85 & 0.30 & 9.0 \\
\hline RXC J0943.0+4700 & $A 0851$ & 145.7600 & 47.0038 & 0.406 & 1.014 & 1.017 & 30.8 & 7.10 & 0.05 & 4.5 \\
\hline RXC J0947.1+5428 & $\ldots$ & 146.7862 & 54.4754 & 0.046 & 5.241 & 15.090 & 2.6 & 0.46 & 0.27 & 14.0 \\
\hline RXC J0952.8+5153 & $\ldots$ & 148.2009 & 51.8888 & 0.214 & 4.196 & 4.216 & 10.6 & 8.13 & 0.21 & 8.0 \\
\hline RXC J0953.6+0142 & $\ldots$ & 148.4231 & 1.7118 & 0.098 & 2.389 & 2.322 & 24.3 & 0.97 & 0.11 & 9.5 \\
\hline RXC J1000.5+4409 & $\ldots$ & 150.1260 & 44.1550 & 0.154 & 2.775 & 2.766 & 12.6 & 2.83 & 0.14 & 5.0 \\
\hline RXC J1013.7-0006 & $\ldots$ & 153.4368 & -0.1085 & 0.093 & 3.382 & 3.353 & 28.4 & 1.25 & 0.16 & 8.0 \\
\hline RXC J1017.5+5933 & $A 0959$ & 154.3960 & 59.5577 & 0.353 & 4.079 & 4.109 & 11.4 & 21.15 & 0.21 & 13.0 \\
\hline RXC J1022.5+5006 & $\ldots$ & 155.6283 & 50.1030 & 0.158 & 5.389 & 5.403 & 9.0 & 5.77 & 0.27 & 8.0 \\
\hline RXC J1023.6+0411 & $\ldots$ & 155.9125 & 4.1873 & 0.285 & 8.562 & 8.617 & 8.1 & 29.21 & 0.42 & 7.5 \\
\hline RXC J1023.6+4908 & $A 0990$ & 155.9212 & 49.1349 & 0.144 & 8.180 & 8.202 & 7.3 & 7.23 & 0.42 & 10.0 \\
\hline RXC J1053.7+5452 & $\ldots$ & 163.4349 & 54.8726 & 0.075 & 4.024 & 3.907 & 11.5 & 0.96 & 0.20 & 11.0 \\
\hline RXC J1058.4+5647 & $\ldots$ & 164.6097 & 56.7922 & 0.136 & 7.661 & 7.682 & 7.0 & 6.09 & 0.40 & 8.5 \\
\hline
\end{tabular}

units of $\mathrm{deg}^{-2}\left(\sigma_{0}\right.$ is not a fit parameter, therefore the error is not provided). Column 3 lists the background galaxy number density around the cluster in units of $\mathrm{deg}^{-2}$. Column 4 shows the core radius estimated from the fit, in units of Mpc. Column 5 provides the cluster total radius, extrapolated from the King profile, in units of Mpc. Column 6 gives the half-light radius in units of Mpc. All the errors in the table are at $68 \%$ confidence level.

The full set of extended tables is available in electronic form.

\section{Correlating X-ray and optical properties}

For a cluster in which mass traces optical light $\left(M / L_{\mathrm{op}}\right.$ is constant), the gas is in hyrostatic equilibrium $\left(T \propto M^{2 / 3}\right)$, and
$L_{\mathrm{X}} \propto T^{3}$ (Xue \& Wu 2000), we expect the X-ray bolometric luminosity to be related to the optical luminosity as $L_{\mathrm{op}} \propto L_{\mathrm{X}}^{0.5}$ and to the intracluster medium temperature as $L_{\mathrm{op}} \propto T_{\mathrm{X}}^{1.5}$.

We now have an optimal data base to test these scaling relations. In a first step we look for those optical parameters which are best suited for a correlation analysis and use these in a second step in the test of the scaling relations.

In this section we show that tight correlations exist between the total optical cluster luminosity and the X-ray cluster properties such as the X-ray luminosity and the intracluster medium temperature.

To search for the best correlation between optical and X-ray properties and to optimally predict for example the X-ray luminosity from the optical appearance, we are interested in an optical characteristic that shows a minimum scatter in the 
Table 2. Example of the table containing optical properties of the whole cluster sample in the $r$ band. The table show the results for the first 35 clusters in the sample.

\begin{tabular}{|c|c|c|c|c|c|c|}
\hline Name & $\alpha$ & $M *$ & $\rho$ & $\chi / v$ & $L_{\mathrm{F}}$ & $L_{\mathrm{C}}$ \\
\hline RXC J0041.8-0918 & $-1.52 \pm 0.18$ & $-22.19 \pm 0.31$ & 2227 & 1.2 & $1.81 \pm 0.22$ & $1.87 \pm 0.06$ \\
\hline RXC J0114.9+0022 & $-1.28 \pm 0.12$ & $-22.01 \pm 0.35$ & 759 & 0.9 & $1.27 \pm 0.18$ & $1.34 \pm 0.04$ \\
\hline RXC J0119.6+1453 & $-1.07 \pm 0.26$ & $-21.92 \pm 0.24$ & 2848 & 0.9 & $4.12 \pm 1.22$ & $2.55 \pm 0.23$ \\
\hline RXC J0137.2-0912 & $-1.78 \pm 0.12$ & $-23.33 \pm 0.33$ & 1036 & 1.5 & $0.69 \pm 0.11$ & $0.70 \pm 0.13$ \\
\hline RXC J0152.7+0100 & $0.00 \pm 0.00$ & $0.00 \pm 0.00$ & 0 & 0 & $0.00 \pm 0.00$ & $3.02 \pm 1.24$ \\
\hline RXC J0736.4+3925 & $-0.87 \pm 0.18$ & $-20.84 \pm 0.42$ & 1274 & 0.8 & $1.06 \pm 0.31$ & $0.82 \pm 0.09$ \\
\hline RXC J0747.0+4131 & $-2.50 \pm 0.00$ & $-18.02 \pm 0.00$ & 263 & 0.8 & $0.12 \pm 0.07$ & $0.00 \pm 0.00$ \\
\hline RXC J0753.3+2922 & $-1.59 \pm 0.11$ & $-22.85 \pm 0.26$ & 1763 & 1.6 & $1.38 \pm 0.20$ & $1.42 \pm 0.08$ \\
\hline RXC J0758.4+3747 & $0.00 \pm 0.00$ & $0.00 \pm 0.00$ & 0 & 0.0 & $0.00 \pm 0.00$ & $0.05 \pm 0.04$ \\
\hline RXC J0800.9+3602 & $-0.90 \pm 0.17$ & $-21.33 \pm 0.43$ & 5523 & 1.2 & $4.81 \pm 2.13$ & $2.62 \pm 0.14$ \\
\hline RXC J0809.6+3455 & $-1.45 \pm 0.17$ & $-21.57 \pm 0.32$ & 2041 & 0.5 & $1.09 \pm 0.18$ & $1.16 \pm 0.10$ \\
\hline RXC J0810.3+4216 & $-1.61 \pm 0.18$ & $-22.56 \pm 0.81$ & 1120 & 0.6 & $0.72 \pm 0.18$ & $0.76 \pm 0.09$ \\
\hline RXC J0822.1+4705 & $-0.71 \pm 0.10$ & $-21.37 \pm 0.76$ & 1422 & 0.8 & $1.52 \pm 0.27$ & $1.49 \pm 0.08$ \\
\hline RXC J0824.0+0326 & $0.00 \pm 0.00$ & $0.00 \pm 0.00$ & 0 & 0 & $0.00 \pm 0.00$ & $0.95 \pm 0.25$ \\
\hline RXC J0825.4+4707 & $-0.71 \pm 0.18$ & $-20.57 \pm$ & 3518 & 0.6 & $2.30 \pm$ & $2.09 \pm$ \\
\hline RXC J0828.1+4445 & $-1.71 \pm 0.14$ & $-23.50 \pm 0.44$ & 2885 & 0.5 & $1.40 \pm 0.36$ & $1.79 \pm 0.21$ \\
\hline RXC J0842.9+3621 & $-1.54 \pm 0.16$ & $-21.71 \pm 0.50$ & 7211 & 0.2 & $2.40 \pm$ & $3.92 \pm 0.27$ \\
\hline RXC J0845.3+4430 & $0.00 \pm 0.00$ & $0.00 \pm 0.00$ & 0 & 0 & $0.00 \pm 0.00$ & $0.17 \pm 0.12$ \\
\hline RXC J0850.1+3603 & $-0.58 \pm 0.18$ & $-21.31 \pm 0.31$ & 8955 & 0.2 & $4.52 \pm 0.67$ & $5.21 \pm 0.17$ \\
\hline RXC J0913.7+4056 & $-0.01 \pm 0.02$ & $-20.69 \pm 0.37$ & 1235 & 0.4 & $1.20 \pm 0.92$ & $0.79 \pm 0.14$ \\
\hline RXC J0913.7+4742 & $-1.29 \pm 0.19$ & $-21.66 \pm 1.34$ & 319 & 0.8 & $0.41 \pm 0.11$ & $0.41 \pm 0.03$ \\
\hline RXC J0917.8+5143 & $-1.07 \pm 0.17$ & $-21.42 \pm 0.27$ & 8128 & 0.6 & $4.02 \pm 0.53$ & $3.86 \pm 0.14$ \\
\hline RXC J0943.0+4700 & $-1.29 \pm 0.11$ & $-21.87 \pm 0.54$ & 6627 & 1.3 & $4.27 \pm 1.01$ & $5.46 \pm 0.30$ \\
\hline RXC J0947.1+5428 & $0.00 \pm 0.00$ & $-20.24 \pm 0.32$ & 64 & 0.6 & $0.40 \pm 0.12$ & $0.34 \pm 0.30$ \\
\hline RXC J0952.8+5153 & $-0.13 \pm 0.20$ & $-20.15 \pm 0.43$ & 2041 & 0.3 & $0.84 \pm 0.24$ & $0.80 \pm 0.05$ \\
\hline RXC J0953.6+0142 & $-1.73 \pm 0.14$ & $-26.00 \pm 0.68$ & 1051 & 0.5 & $1.18 \pm 0.60$ & $1.16 \pm 0.07$ \\
\hline RXC J1000.5+4409 & $-1.35 \pm 1.05$ & $-22.11 \pm 0.28$ & 542 & 0.4 & $0.34 \pm 0.19$ & $0.31 \pm 0.08$ \\
\hline RXC J1013.7-0006 & $-1.05 \pm 0.14$ & $-20.80 \pm 0.31$ & 1324 & 0.6 & $1.00 \pm 0.22$ & $0.85 \pm 0.16$ \\
\hline RXC J1017.5+5933 & $-1.44 \pm 0.18$ & $-23.07 \pm 0.47$ & 6321 & 1.6 & $4.40 \pm 0.87$ & $5.53 \pm 0.30$ \\
\hline RXC J1022.5+5006 & $-1.19 \pm 0.10$ & $-21.55 \pm 0.38$ & 4271 & 0.7 & $2.38 \pm 0.36$ & $2.25 \pm 0.10$ \\
\hline RXC J1023.6+0411 & $-0.98 \pm 0.15$ & $-20.81 \pm 0.25$ & 5696 & 0.1 & $1.97 \pm 0.44$ & $2.25 \pm 0.12$ \\
\hline RXC J1023.6+4908 & $-0.84 \pm 0.10$ & $-20.85 \pm 0.25$ & 2664 & 0.4 & $1.65 \pm 0.32$ & $1.46 \pm 0.06$ \\
\hline RXC J1053.7+5452 & $-1.43 \pm 0.17$ & $-22.19 \pm 0.37$ & 1320 & 0.8 & $1.06 \pm 0.17$ & $1.08 \pm 0.05$ \\
\hline RXC J1058.4+5647 & $-1.59 \pm 0.25$ & $-22.26 \pm 0.23$ & 4433 & 1.1 & $2.15 \pm 0.42$ & $2.15 \pm 0.28$ \\
\hline
\end{tabular}

X-ray/optical correlation. Therefore, we perform a correlation using 4 of the 5 SDSS optical band, $g, r, i$ and $z$, to find out which filter should be used in the prediction. The $u$ band was not used since the cluster $\mathrm{S} / \mathrm{N}$ in that band is too low to calculate the cluster total luminosity. We used a fixed aperture to calculate the optical luminosities for all the clusters, in order to make no a priori assumption about the cluster size. Moreover, to check whether the scatter in the correlation depends on the cluster aperture, we did the same analysis several times using optical luminosities calculated within different radii, ranging from 0.05 to $4 \mathrm{Mpc} h_{70}^{-1}$ from the X-ray center. To quantify the $L_{\mathrm{op}}-L_{\mathrm{X}}$ and the $L_{\mathrm{op}}-T_{\mathrm{X}}$ relations, a linear regression in loglog space was performed using two methods for the fitting: a numerical orthogonal distance regression method (ODRPACK) and the bisector method (Akritas \& Bershady 1996). The fits are performed using the form

$\log \left(L_{\mathrm{op}} / L_{\odot}\right)=\alpha \log \left(P_{\mathrm{X}}\right)+\beta$ where $P_{\mathrm{X}}$ is the $\mathrm{X}$-ray property, and the errors of each variable are transformed into $\log$ space as $\Delta \log (x)=\log (e)\left(x^{+}-\right.$ $\left.x^{-}\right) /(2 x)$, where $x^{+}$and $x^{-}$denote the upper and lower boundary of the error range of the quantity, respectively. To exclude the outliers in the fitting procedure we apply a $\sigma$ clipping method. After a first fit all the points featuring a larger than $3 \sigma$ deviation from the relation, were excluded and the fitting procedure was repeated (see discussion below).

Figures 15 and 16 show the scatter of the $L_{\mathrm{op}}-L_{\mathrm{X}}$ and the $L_{\mathrm{op}}-T_{\mathrm{X}}$ relations, respectively, versus the cluster aperture used to calculate the optical luminosity. The scatter in the plot is the orthogonal scatter estimated from the best fit given by ODRPACK. In any photometric band the scatter has a clear dependence on the cluster aperture showing a minimum in the very center of the cluster, between $0.2 \mathrm{Mpc} h_{70}^{-1}$ and $0.8 \mathrm{Mpc} h_{70}^{-1}$, and increasing at larger radii. The scatter is partially due to the method used to calculate the optical luminosity. Our method is simply based on the overdensity of the 
Table 3. Example of the table containing the optical properties of the whole cluster sample in the $r$ band. The table shows the results for the first 35 cluster of the sample.

\begin{tabular}{|c|c|c|c|c|c|c|}
\hline Name & $\bar{\beta}$ & $\overline{\sigma_{0}}$ & $\overline{\sigma_{\mathrm{b}}}$ & $\overline{r_{\mathrm{c}}}$ & $\overline{r_{\mathrm{tot}}}$ & $\overline{r_{h}}$ \\
\hline RXC J0041.8-0918 & $0.88 \pm 0.16$ & 5731 & $2295 \pm 456$ & $1.15 \pm 0.04$ & 2.33 & $1.00 \pm 0.37$ \\
\hline RXC J0114.9+0022 & $1.61 \pm 0.48$ & 2281 & $4540 \pm 106$ & $51 \pm 0.07$ & 0.78 & $0.36 \pm 0.09$ \\
\hline RXC J0119.6+1453 & $0.44 \pm 0.14$ & 17803 & $4051 \pm 896$ & $0.08 \pm 0.04$ & 0.67 & $0.22 \pm 0.16$ \\
\hline RXC J0137.2-0912 & $1.68 \pm 0.33$ & 4955 & $5256 \pm 37$ & $0.20 \pm 0.03$ & 0.59 & $0.22 \pm 0.14$ \\
\hline RXC J0152.7+0100 & $0.78 \pm 0.21$ & 12846 & $4297 \pm 607$ & $0.40 \pm 0.07$ & 1.34 & $0.87 \pm 0.16$ \\
\hline RXC J0736.4+3925 & $1.49 \pm 0.40$ & 4834 & $2083 \pm 594$ & $0.57 \pm 0.12$ & 2.52 & $1.27 \pm 0.26$ \\
\hline RXC J0747.0+4131 & $0.00 \pm 0.00$ & 0 & $0 \pm 0$ & $0.00 \pm 0.00$ & 0.00 & $0.00 \pm 0.00$ \\
\hline RXC J0753.3+2922 & $1.06 \pm 0.25$ & 5076 & $4314 \pm 270$ & $0.48 \pm 0.05$ & 1.03 & $0.43 \pm 0.10$ \\
\hline RXC J0758.4+3747 & $0.00 \pm 0.00$ & 0 & $0 \pm 0$ & $0.00 \pm 0.00$ & 0.00 & $0.00 \pm 0.00$ \\
\hline RXC J0800.9+3602 & $0.40 \pm 0.131$ & 30806 & $3649 \pm 1807$ & $0.11 \pm 0.05$ & 0.96 & $0.75 \pm 0.35$ \\
\hline RXC J0809.6+3455 & $0.18 \pm 0.212$ & 23059 & $3860 \pm 2779$ & $0.00 \pm 0.03$ & 0.02 & $0.00 \pm 0.00$ \\
\hline RXC J0810.3+4216 & $0.46 \pm 0.16$ & 9392 & $3998 \pm 536$ & $0.07 \pm 0.03$ & 0.48 & $0.36 \pm 0.14$ \\
\hline RXC J0822.1+4705 & $0.83 \pm 0.20$ & 8734 & $3817 \pm 262$ & $0.26 \pm 0.05$ & 1.09 & $0.52 \pm 0.13$ \\
\hline RXC J0824.0+0326 & $0.76 \pm 0.26$ & 32273 & $5369 \pm 588$ & $0.08 \pm 0.04$ & 0.56 & $0.23 \pm 0.24$ \\
\hline RXC J0825.4+4707 & $0.90 \pm 0.13$ & 15229 & $4130 \pm 292$ & $0.32 \pm 0.03$ & 1.54 & $0.64 \pm 0.18$ \\
\hline RXC J0828.1+4445 & $1.12 \pm 0.13$ & 46498 & $5262 \pm 108$ & $0.11 \pm 0.02$ & 1.02 & $0.42 \pm 0.14$ \\
\hline RXC J0842.9+3621 & $0.54 \pm 0.08$ & 50832 & $4247 \pm 606$ & $0.09 \pm 0.03$ & 1.96 & $1.57 \pm 0.21$ \\
\hline RXC J0845.3+4430 & $0.59 \pm 0.51$ & 6690 & $3060 \pm 974$ & $0.04 \pm 0.03$ & 0.07 & $0.00 \pm 0.00$ \\
\hline RXC J0850.1+3603 & 2.05 & 24289 & $6445 \pm$ & $4 \pm 0.06$ & 1.50 & 0.31 \\
\hline RXC J0913.7+4056 & $0.62 \pm 0.21$ & 31255 & $3200 \pm 581$ & $0.08 \pm 0.05$ & 0.84 & $0.31 \pm 0.13$ \\
\hline RXC J0913.7+4742 & $1.23 \pm 0.43$ & 3911 & $4558 \pm 65$ & $0.15 \pm 0.04$ & 0.48 & $0.32 \pm 0.10$ \\
\hline RXC J0917.8+5143 & $0.89 \pm 0.09$ & 43660 & $4508 \pm 308$ & $0.23 \pm 0.03$ & 2.01 & $0.55 \pm 0.23$ \\
\hline RXC J0943.0+4700 & $0.46 \pm 0.111$ & 54863 & $2903 \pm 1315$ & $0.07 \pm 0.04$ & 1.21 & $0.65 \pm 0.18$ \\
\hline RXC J0947.1+5428 & $0.00 \pm 0.00$ & 0 & $0 \pm 0$ & $0.00 \pm 0.00$ & 0.00 & $0.00 \pm 0.00$ \\
\hline RXC J0952.8+5153 & $0.94 \pm 0.18$ & 16193 & $3828 \pm 207$ & $0.21 \pm 0.05$ & 1.16 & $0.58 \pm 0.20$ \\
\hline RXC J0953.6+0142 & $1.30 \pm 0.55$ & 4345 & $4761 \pm 157$ & $0.28 \pm 0.07$ & 0.59 & $0.28 \pm 0.12$ \\
\hline RXC J1000.5+4409 & $0.81 \pm 0.16$ & 20092 & $3245 \pm 199$ & $0.10 \pm 0.03$ & 0.88 & $0.27 \pm 0.14$ \\
\hline RXC J1013.7-0006 & $0.77 \pm 0.13$ & 16004 & $4101 \pm 223$ & $0.11 \pm 0.03$ & 0.90 & $0.22 \pm 0.19$ \\
\hline RXC J1017.5+5933 & $1.21 \pm 0.13$ & 44927 & $3836 \pm 193$ & $0.30 \pm 0.04$ & 1.77 & $0.71 \pm 0.27$ \\
\hline RXC J1022.5+5006 & $0.79 \pm 0.10$ & 33560 & $4690 \pm 263$ & $0.14 \pm 0.02$ & 1.45 & $0.55 \pm 0.20$ \\
\hline RXC J1023.6+0411 & $0.55 \pm 0.07$ & 97158 & $4280 \pm 429$ & $0.04 \pm 0.02$ & 2.07 & $1.49 \pm 0.72$ \\
\hline RXC J1023.6+4908 & $0.91 \pm 0.14$ & 14311 & $3679 \pm 222$ & $0.28 \pm 0.04$ & 1.50 & $0.33 \pm 0.09$ \\
\hline RXC J1053.7+5452 & $3.00 \pm 0.00$ & 2855 & $5201 \pm 41$ & $0.60 \pm 0.09$ & 0.82 & $0.34 \pm 0.11$ \\
\hline RXC J1058.4+5647 & $1.14 \pm 0.12$ & 24980 & $4836 \pm 151$ & $0.26 \pm 0.03$ & 1.48 & $0.91 \pm 0.22$ \\
\hline
\end{tabular}

cluster with respect of the galaxy background. In fact if the central region in which $L_{\mathrm{op}}$ is measured is too small $\left(0.05 \mathrm{Mpc} h_{70}^{-1}\right.$ in Figs. 15 and 16), the value of the galaxy density is low and the measurement becomes more uncertain. On the other hand, at larger radii the density contrast between cluster and background decreases progressively. Instead, within a cluster aperture between $0.2 \mathrm{Mpc} h_{70}^{-1}$ and $0.8 \mathrm{Mpc} h_{70}^{-1}$, the optical luminosity of both groups and massive clusters can be easily measured. In fact in both cases the radial aperture is small enough to show an high density contrast and therefore a high cluster $\mathrm{S} / \mathrm{N}$, and still large to enclose enough galaxies for the luminosity calculation.

After a more accurate analysis, we noted also that the low luminosity systems (both in the optical and in the X-ray band) are the main source of scatter at any cluster aperture. This could be due to different reasons. From the technical point of view the groups have a lower surface density contrast, and this causes problems in calculating the optical luminosity with a method based on the overdensity contrast. Moreover, the low mass systems could have a larger scatter in the optical and $\mathrm{X}$-ray properties.

Furthermore, the galaxy groups could be responsible for the behavior of the scatter shown in Fig. 15. In fact at large cluster apertures the galaxy density contrast can be very low for the small systems and still very high for the massive and larger clusters. The large error introduced by the low density contrast in the calculation of the optical luminosity of galaxy groups could explain the increment of the scatter at larger apertures. To study in more detail the nature of the scatter of our correlation, and to investigate the role of the less luminous systems, we carried out the analysis explained above with the low mass systems removed. We limited the analysis to the subsample of the X-ray selected REFLEX-NORAS clusters, which occupy the intermediate and high luminosity region. Figure 17 shows the behavior of the scatter as a function of the cluster aperture in this second analysis. After removing the low mass systems the 


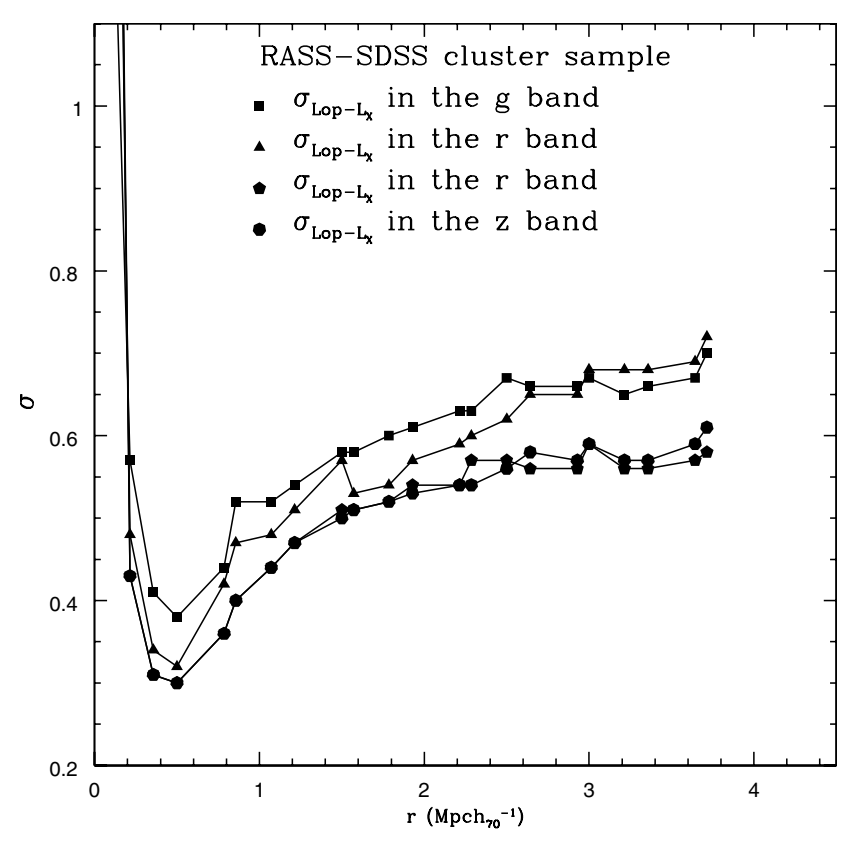

Fig. 15. Orthogonal scatter to the best fit of the $L_{\mathrm{op}}-L_{\mathrm{X}}$ relation obtained with the orthogonal method, as a function of the cluster aperture. The scatter shows a minimum in the region $0.2 \leq r \leq$ $0.8 \mathrm{Mpc} h_{70}^{-1}$. Different symbols relate to the different photometric bands in which $L_{\mathrm{op}}$ is calculated: squares for the $g$ band, triangles for the $r$ band, hexagons for $i$ band and filled circles for the $z$ band. The $i$ and $z$ bands clearly show the smallest scatter at any aperture.

scatter decreases by about $30 \%$ for any aperture (from 0.3 dex to 0.2 dex in the region of minimum scatter and from $0.6 \mathrm{dex}$ on average to 0.4 dex at larger apertures). Nevertheless the behavior of the scatter at increasing aperture is exactly the same observed in the analysis carried out on the overall RASS-SDSS galaxy cluster sample. This means that groups are responsible for part of the scatter but cannot explain the existence of the region of minimum scatter between 0.2 and $0.8 \mathrm{Mpc} h_{70}^{-1}$. A possible explanation for the behavior of the scatter at different cluster apertures could be the cluster compactness. As $L_{X}$ depends not only on the cluster mass but also on the compactness of the cluster, the optical luminosity should also reflect somehow the cluster properties, mass and concentration. Thus, there should be an optimal aperture radius within which to measure $L_{\mathrm{op}}$. We found that in all photometric bands, the minimum scatter is around $0.5 \mathrm{Mpc} h_{70}^{-1}$.

Figures 18 and 19 show the $L_{\mathrm{op}}-L_{\mathrm{X}}$ and $L_{\mathrm{op}}-T_{\mathrm{X}}$ relation respectively, at the radius of minimum scatter, $0.5 \mathrm{Mpc} h_{70}^{-1}$, in the $z$ band. In fact the $i$ and the $z$ bands have a slightly smaller scatter than the other optical bands at any radius. Both plots show, as an outlier, the cluster RXC J0845.3+4430, which features a deviation larger than $3 \sigma$ from the best fit. The system is a nearby group with a density contrast too low to estimate the optical luminosity reliably. The X-ray luminosity of this system has a $100 \%$ error. In $L_{\mathrm{op}}-T_{\mathrm{X}}$ there is another outlier: the cluster RXC J1629.6+4049. This system is not a source of scatter in the $L_{\mathrm{op}}-L_{\mathrm{X}}$ relation and the error in the X-ray and optical luminosities is $8 \%$ and $35 \%$ respectively. This can suggests that the optical luminosity is well measured, and it questions the estimate of the temperature. In fact, the X-ray luminosity

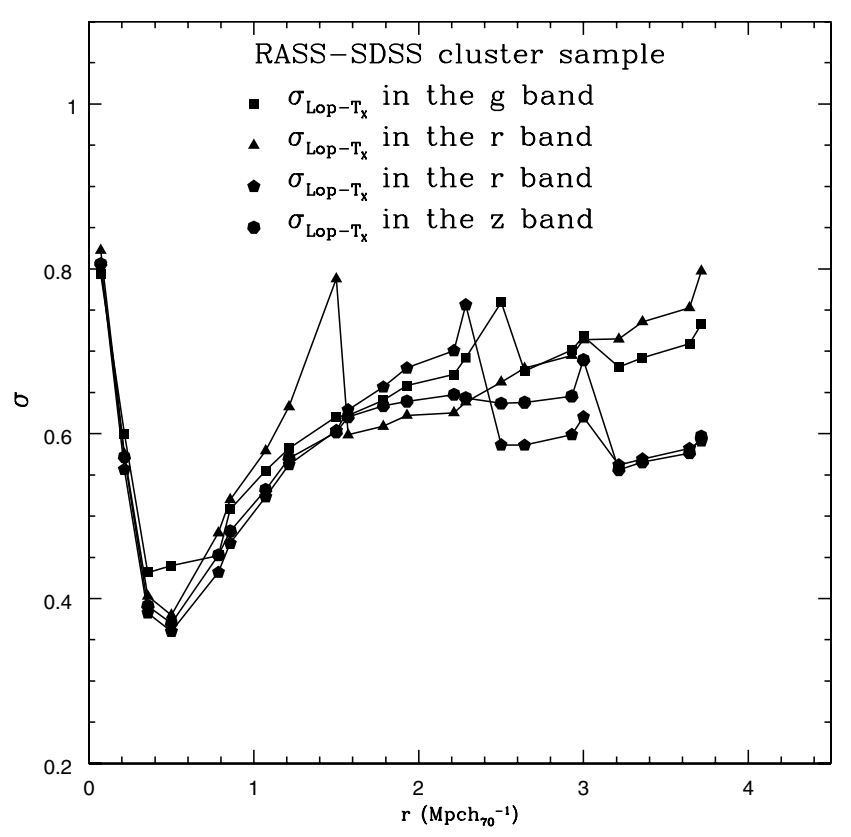

Fig. 16. Orthogonal scatter to the best fit of the $L_{\mathrm{op}}-T_{\mathrm{X}}$ relation obtained with the orthogonal method, as a function of the cluster aperture. The scatter shows a minimum in the region $0.2 \leq r \leq$ $0.8 \mathrm{Mpc} h_{70}^{-1}$. Different symbols relate to the different photometric bands in which $L_{\mathrm{op}}$ is calculated: squares for the $g$ band, triangles for the $r$ band, hexagons for $i$ band and filled circles for the $z$ band. The $i$ and $z$ bands clearly show the smallest scatter at any aperture.

of RXC J1629.6+4049 is $2.78 \times 10^{43} \mathrm{erg} \mathrm{s}^{-1}$, and the temperature, estimated from Horner et al. (2001), is $1 \mathrm{keV}$, while the $L_{\mathrm{X}}-T_{\mathrm{X}}$ relation of Xue $\& \mathrm{Wu}(2000)$, predicts at least a $T_{\mathrm{X}}$ of $4.3 \mathrm{keV}$ at that $L_{\mathrm{X}}$.

With the $\sigma$ clipping method those clusters were excluded from the estimation of the best fit. The best fit parameters of the orthogonal and bisector methods, in the $i$ and $z$ photometric bands, are shown in Tables 4 and 5 for the $L_{\mathrm{op}}-L_{\mathrm{X}}$ relation for the ROSAT X-ray luminosity and the bolometric X-ray luminosity, respectively. Table 6 shows the same results for the $L_{\mathrm{op}}-T_{\mathrm{X}}$ relation. Table 7 provides the results for the $L_{\mathrm{op}}-L_{\mathrm{X}}$ relation in the $i$ and $z$ band for the subsample of X-ray selected REFLEX-NORAS clusters. The tables also give the estimated orthogonal scatter and the estimated scatter in the two variables. The best fits in the $z$ band for the $L_{\mathrm{op}}-L_{\mathrm{X}}$ and the $L_{\mathrm{op}}-T_{\mathrm{X}}$ relations at the radius of minimum scatter for the whole RASS-SDSS galaxy cluster sample are respectively:

$$
\begin{aligned}
& L_{\mathrm{op}} / L_{\odot}=10^{11.79 \pm 0.02} L_{\mathrm{X}}(\operatorname{ROS} A T)^{0.45 \pm 0.03} \\
& L_{\mathrm{op}} / L_{\odot}=10^{11.75 \pm 0.02} L_{\mathrm{X}}(\mathrm{Bol})^{0.38 \pm 0.02} \\
& L_{\mathrm{op}} / L_{\odot}=10^{11.42 \pm 0.06} T_{\mathrm{X}}^{1.12 \pm 0.08}
\end{aligned}
$$

The value of the exponent in the power law for the $L_{\mathrm{op}}-L_{\mathrm{X}}(\mathrm{Bol})$ relation is around 0.38 in the region of minimum, as indicated in Table 4. The values are not consistent within the errors with the value of 0.5 predicted under the assumption of hydrostatic equilibrium and constant mass to light ratio. The same conclusion can be reached for the $L_{\mathrm{op}}-T_{\mathrm{X}}$ relation and from 


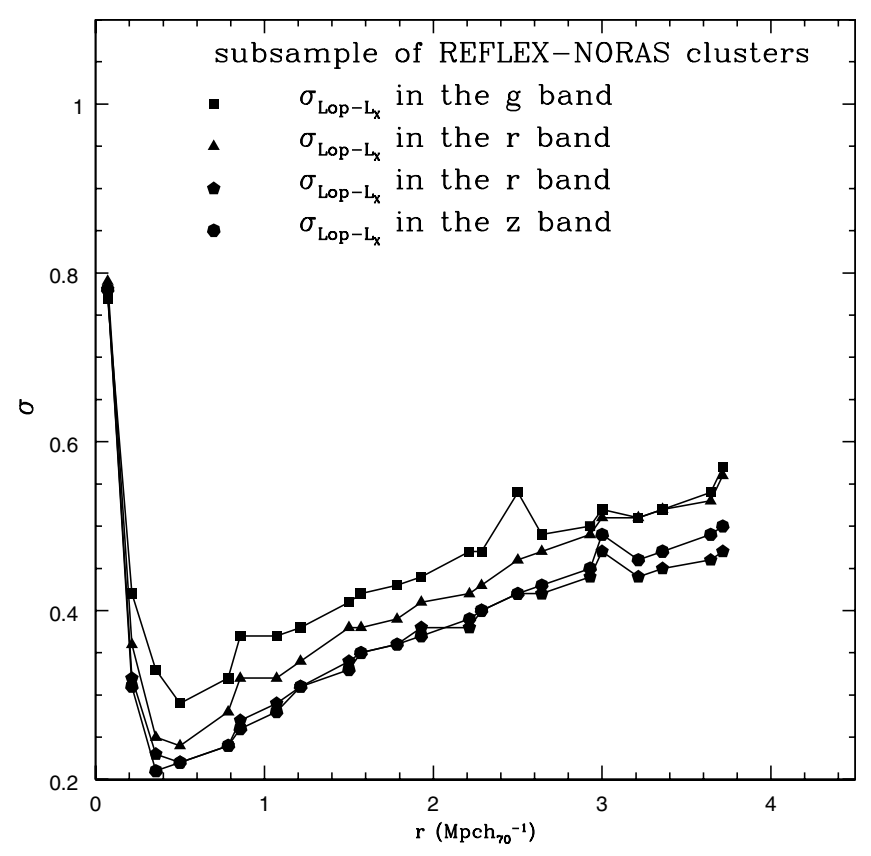

Fig. 17. Orthogonal scatter to the best fit of the $L_{\mathrm{op}}-L_{\mathrm{X}}$ relation obtained with the orthogonal method, as a function of the cluster aperture for the subsample of REFLEX - NORAS clusters. The scatter shows again a minimum in the region $0.2 \leq r \leq 0.8 \mathrm{Mpc} h_{70}^{-1}$. Different symbols relate to the different photometric bands in which $L_{\mathrm{op}}$ is calculated: squares for the $g$ band, triangles for the $r$ band, hexagons for $i$ band and filled circles for the $z$ band. The $i$ and $z$ bands clearly show the smallest scatter at any aperture.

the $L_{\mathrm{op}}-L_{\mathrm{X}}$ for the subsample of X-ray selected REFLEXNORAS clusters. A simple reason for the disagreement could be the assumption of a constant mass to light ratio. In fact, Girardi et al. (2002) analysed in detail the mass to light ratio in the $B$ band of a sample of 294 clusters and groups, finding $M / L \propto L^{0.33 \pm 0.03}$. The same result was found by Lin et al. (2003) in the $K$ band. Thus if we combine this dependence of $M / L$ from the optical luminosity with the assumptions of hydrostatic equilibrium, the new expected relations between the optical luminosity and the X-ray luminosity and temperature are $L_{\mathrm{op}} \propto L_{\mathrm{X}}^{0.4}$ and $L_{\mathrm{op}} \propto T_{\mathrm{X}}^{1.25}$, respectively, which are in good agreement with our results.

The scatter in the $L_{\mathrm{op}}-L_{\mathrm{X}}$ relation for the aperture with the best correlation $\left(0.5 \mathrm{Mpc} h_{70}^{-1}\right)$, in the $L_{\mathrm{op}}$ variable is 0.20 , and the scatter in the $L_{\mathrm{X}}$ variable is 0.22 in the correlations obtained in the $i$ and $z$ bands as shown in Table 4. Therefore, by calculating the total cluster luminosity in the central part of the system, one can use the $i$ or $z$ band to predict the X-ray luminosity from the optical data with a mean error of $60 \%$. In the same way the optical luminosity can be derived from $L_{X}$ with the same uncertainty. As indicated in Table 7, the uncertainty in the prediction of the two variables decreases to less than $40 \%$ if the correlation is limited to the REFLEX-NORAS cluster subsample. Table 6 shows that analogous results are obtained for the $L_{\mathrm{op}}-T_{\mathrm{X}}$ relation.

Since the observational uncertainties in the optical and in the X-ray luminosity are about $20 \%$, the scatter of $60 \%$ of the

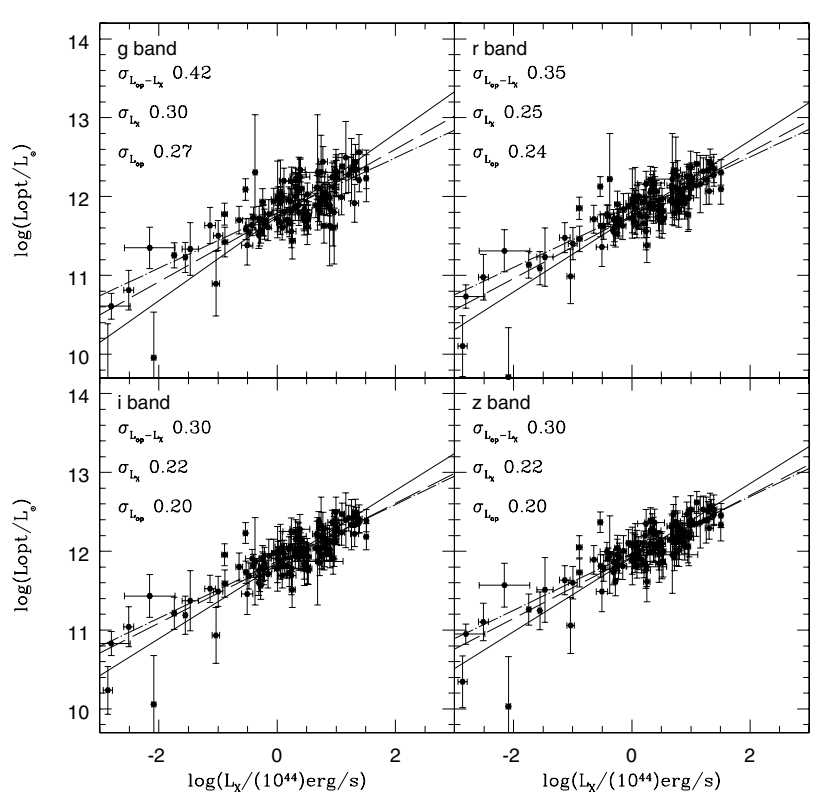

Fig. 18. Correlation between optical luminosities and X-ray luminosities. The fit is performed with a linear regression in the $\log \left(L_{\mathrm{op}}\right)-$ $\log \left(L_{\mathrm{X}}\right)$ space for each of the 4 optical bands. The solid and the dashed lines are the results of the orthogonal and the bisector method respectively over the whole RASS-SDSS galaxy cluster sample. The dotdashed line is the best fit result of the orthogonal method applied to the subsample of strictly X-ray selected REFLEX-NORAS clusters. The error bars are at the $68 \%$ confidence level in both variables.

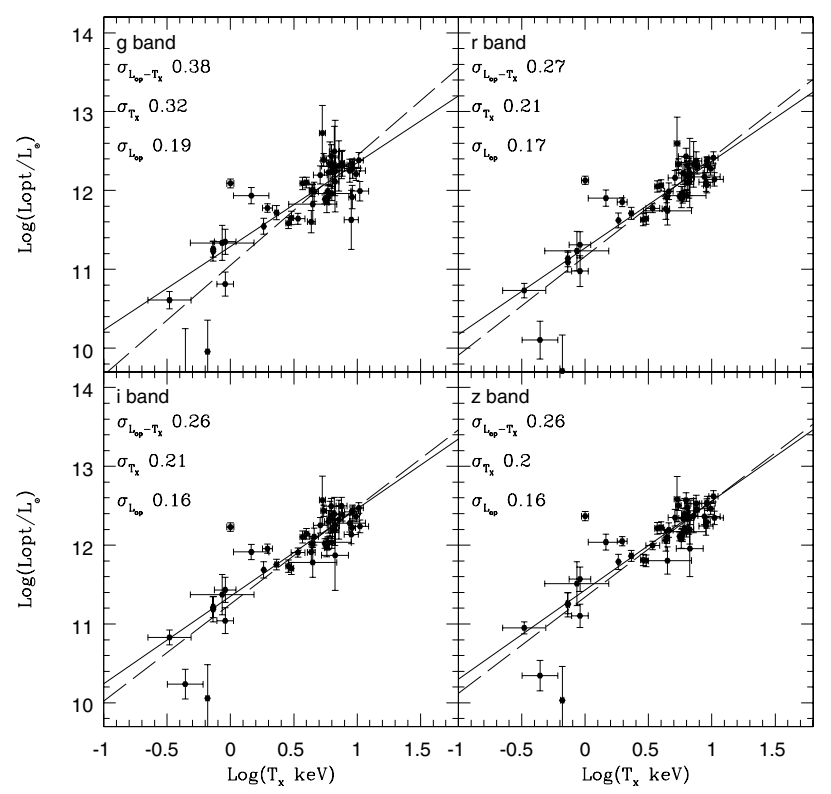

Fig. 19. Correlation between optical luminosities and ICM temperature. The fit is performed with a linear regression in the $\log \left(L_{\mathrm{op}}\right)-$ $\log \left(T_{\mathrm{X}}\right)$ space for each of the 4 optical bands. The solid and the dashed lines are the results of the orthogonal and the bisector method respectively. The error bars are at the $68 \%$ confidence level in both variables.

overall sample in both relations and of $40 \%$ in the REFLEXNORAS cluster subsample for the $L_{\mathrm{op}}-L_{\mathrm{X}}$ relation should be intrinsic. 
Table 4. The table presents the results of the best fit for the $i$ and $z$ bands in the region of minimum scatter, $0.2 \leq r \leq 1.0 \mathrm{Mpc} h_{70}^{-1}$ for the $L_{\mathrm{op}}-L_{\mathrm{X}}(R O S A T)$ relation. We show the results for the two methods applied: the orthogonal distance regression (ODRPACK) and the bisector method, which is the line bisecting the best fit results of the vertical and horizontal minimization. The $\alpha$ and $\beta$ parameters are given for several apertures with $95 \%$ confidence errors. The orthogonal scatter and the scatter in $\log \left(L_{X}(0.1-2.4 \mathrm{keV})\right)$ and in $\log \left(L_{\mathrm{op}}\right)$ to the best fit line are given by $\sigma, \sigma_{L_{X}}$, and $\sigma_{L_{\mathrm{op}}}$, respectively.

\begin{tabular}{|c|c|c|c|c|c|c|c|c|c|c|}
\hline \multicolumn{11}{|c|}{$L_{\mathrm{op}}-L_{\mathrm{X}}(R O S A T)$ relation in the $I$ band } \\
\hline \multicolumn{6}{|c|}{ Orthogonal method } & \multicolumn{5}{|c|}{ Bisector method } \\
\hline$r$ & $\alpha$ & $\beta$ & $\sigma$ & $\sigma_{L_{\mathrm{X}}}$ & $\sigma_{L_{\mathrm{op}}}$ & $\alpha$ & $\beta$ & $\sigma$ & $\sigma_{L_{X}}$ & $\sigma_{L_{\mathrm{op}}}$ \\
\hline 0.2 & $0.50 \pm 0.04$ & $11.41 \pm 0.03$ & 0.43 & 0.32 & 0.30 & $0.32 \pm 0.01$ & $11.44 \pm 0.03$ & 0.36 & 0.23 & 0.28 \\
\hline 0.3 & $0.46 \pm 0.03$ & $11.70 \pm 0.02$ & 0.31 & 0.23 & 0.21 & $0.36 \pm 0.01$ & $11.71 \pm 0.02$ & 0.29 & 0.18 & 0.23 \\
\hline 0.5 & $0.47 \pm 0.03$ & $11.83 \pm 0.02$ & 0.30 & 0.22 & 0.20 & $0.38 \pm 0.01$ & $11.85 \pm 0.02$ & 0.27 & 0.18 & 0.20 \\
\hline 0.7 & $0.56 \pm 0.03$ & $11.94 \pm 0.03$ & 0.36 & 0.26 & 0.25 & $0.47 \pm 0.01$ & $11.96 \pm 0.02$ & 0.32 & 0.22 & 0.24 \\
\hline 0.8 & $0.61 \pm 0.04$ & $11.99 \pm 0.03$ & 0.40 & 0.29 & 0.28 & $0.51 \pm 0.01$ & $12.01 \pm 0.03$ & 0.36 & 0.24 & 0.26 \\
\hline 1.0 & $0.65 \pm 0.04$ & $12.05 \pm 0.03$ & 0.44 & 0.31 & 0.31 & $0.54 \pm 0.01$ & $12.07 \pm 0.03$ & 0.39 & 0.27 & 0.28 \\
\hline \multicolumn{11}{|c|}{$L_{\mathrm{op}}-L_{\mathrm{X}}(R O S A T)$ relation in the $Z$ band } \\
\hline \multicolumn{6}{|c|}{ Orthogonal method } & \multicolumn{5}{|c|}{ Bisector method } \\
\hline$r$ & $\alpha$ & $\beta$ & $\sigma$ & $\sigma_{L_{X}}$ & $\sigma_{L_{\mathrm{op}}}$ & $\alpha$ & $\beta$ & $\sigma$ & $\sigma_{L_{X}}$ & $\sigma_{L_{\mathrm{op}}}$ \\
\hline 0.2 & $0.50 \pm 0.04$ & $11.50 \pm 0.03$ & 0.43 & 0.31 & 0.31 & $0.31 \pm 0.01$ & $11.54 \pm 0.03$ & 0.35 & 0.23 & 0.26 \\
\hline 0.3 & $0.45 \pm 0.03$ & $11.79 \pm 0.02$ & 0.31 & 0.23 & 0.21 & $0.37 \pm 0.01$ & $11.80 \pm 0.02$ & 0.29 & 0.18 & 0.23 \\
\hline 0.5 & $0.47 \pm 0.03$ & $11.92 \pm 0.02$ & 0.30 & 0.22 & 0.20 & $0.39 \pm 0.01$ & $11.93 \pm 0.02$ & 0.28 & 0.18 & 0.21 \\
\hline 0.7 & $0.56 \pm 0.03$ & $12.03 \pm 0.03$ & 0.36 & 0.26 & 0.25 & $0.47 \pm 0.01$ & $12.04 \pm 0.02$ & 0.33 & 0.22 & 0.24 \\
\hline 0.8 & $0.60 \pm 0.04$ & $12.08 \pm 0.03$ & 0.40 & 0.29 & 0.27 & $0.51 \pm 0.01$ & $12.10 \pm 0.03$ & 0.36 & 0.24 & 0.26 \\
\hline 1.0 & $0.65 \pm 0.04$ & $12.14 \pm 0.03$ & 0.44 & 0.31 & 0.31 & $0.54 \pm 0.01$ & $12.15 \pm 0.03$ & 0.39 & 0.27 & 0.28 \\
\hline
\end{tabular}

Table 5. The table presents the results of the best fit for the $i$ and $z$ bands in the region of minimum scatter, $0.2 \leq r \leq 1.0 \mathrm{Mpc} h_{70}^{-1}$ for the $L_{\mathrm{op}}-L_{\mathrm{X}}$ (Bolometric) relation. We show the results for the two methods applied: the orthogonal distance regression (ODRPACK) and the bisector method, which is the line bisecting the best fit results of the vertical and horizontal minimization. The $\alpha$ and $\beta$ parameters are given for several apertures with $95 \%$ confidence errors. The orthogonal scatter and the scatters in $\log \left(T_{\mathrm{X}}\right)$ and in $\log \left(L_{\mathrm{op}}\right)$ to the best fit line are given by $\sigma, \sigma_{T_{\mathrm{X}}}$, and $\sigma_{L_{\mathrm{op}}}$, respectively.

\begin{tabular}{|c|c|c|c|c|c|c|c|c|c|c|}
\hline \multicolumn{11}{|c|}{$L_{\mathrm{op}}-L_{\mathrm{X}}($ Bolometric $)$ relation in the $I$ band } \\
\hline \multicolumn{6}{|c|}{ Orthogonal method } & \multicolumn{5}{|c|}{ Bisector method } \\
\hline$r$ & $\alpha$ & $\beta$ & $\sigma$ & $\sigma_{L_{X}}$ & $\sigma_{L_{\mathrm{op}}}$ & $\alpha$ & $\beta$ & $\sigma$ & $\sigma_{L_{X}}$ & $\sigma_{L_{\mathrm{op}}}$ \\
\hline 0.2 & $0.38 \pm 0.03$ & $11.25 \pm 0.04$ & 0.41 & 0.31 & 0.27 & $0.27 \pm 0.01$ & $11.31 \pm 0.03$ & 0.37 & 0.24 & 0.28 \\
\hline 0.3 & $0.36 \pm 0.02$ & $11.54 \pm 0.03$ & 0.31 & 0.23 & 0.20 & $0.30 \pm 0.01$ & $11.57 \pm 0.02$ & 0.29 & 0.18 & 0.23 \\
\hline 0.5 & $0.38 \pm 0.02$ & $11.66 \pm 0.03$ & 0.30 & 0.22 & 0.20 & $0.32 \pm 0.01$ & $11.70 \pm 0.02$ & 0.27 & 0.18 & 0.21 \\
\hline 0.7 & $0.38 \pm 0.02$ & $11.66 \pm 0.03$ & 0.30 & 0.22 & 0.20 & $0.32 \pm 0.01$ & $11.70 \pm 0.02$ & 0.27 & 0.18 & 0.21 \\
\hline 0.8 & $0.49 \pm 0.03$ & $11.77 \pm 0.04$ & 0.41 & 0.30 & 0.28 & $0.42 \pm 0.01$ & $11.82 \pm 0.03$ & 0.37 & 0.25 & 0.27 \\
\hline 1.0 & $0.53 \pm 0.03$ & $11.81 \pm 0.04$ & 0.44 & 0.32 & 0.31 & $0.45 \pm 0.01$ & $11.86 \pm 0.03$ & 0.40 & 0.27 & 0.29 \\
\hline \multicolumn{11}{|c|}{$L_{\mathrm{op}}-L_{\mathrm{X}}($ Bolometric $)$ relation in the $Z$ band } \\
\hline \multicolumn{6}{|c|}{ Orthogonal method } & \multicolumn{5}{|c|}{ Bisector method } \\
\hline$r$ & $\alpha$ & $\beta$ & $\sigma$ & ${ }^{0} L_{X}$ & $\sigma_{L_{\mathrm{op}}}$ & $\alpha$ & $\beta$ & $\sigma$ & $\sigma_{L_{X}}$ & $\sigma_{L_{\mathrm{op}}}$ \\
\hline 0.2 & $0.38 \pm 0.03$ & $11.34 \pm 0.04$ & 0.41 & 0.30 & 0.28 & $0.26 \pm 0.01$ & $11.42 \pm 0.03$ & 0.35 & 0.23 & 0.27 \\
\hline 0.3 & $0.35 \pm 0.02$ & $11.63 \pm 0.03$ & 0.30 & 0.24 & 0.19 & $0.31 \pm 0.01$ & $11.65 \pm 0.02$ & 0.29 & 0.18 & 0.23 \\
\hline 0.5 & $0.38 \pm 0.02$ & $11.75 \pm 0.03$ & 0.30 & 0.23 & 0.20 & $0.32 \pm 0.01$ & $11.78 \pm 0.02$ & 0.28 & 0.18 & 0.21 \\
\hline 0.7 & $0.38 \pm 0.02$ & $11.75 \pm 0.03$ & 0.30 & 0.23 & 0.20 & $0.32 \pm 0.01$ & $11.78 \pm 0.02$ & 0.28 & 0.18 & 0.21 \\
\hline 0.8 & $0.49 \pm 0.03$ & $11.86 \pm 0.04$ & 0.40 & 0.29 & 0.28 & $0.42 \pm 0.01$ & $11.90 \pm 0.03$ & 0.37 & 0.25 & 0.27 \\
\hline 1.0 & $0.52 \pm 0.03$ & $11.90 \pm 0.04$ & 0.44 & 0.32 & 0.31 & $0.44 \pm 0.01$ & $11.95 \pm 0.03$ & 0.40 & 0.28 & 0.29 \\
\hline
\end{tabular}

\section{Summary and conclusions}

We created a database of clusters of galaxies based on the largest available X-ray and optical surveys: the ROSAT All Sky Survey (RASS) and the Sloan Digital Sky Survey (SDSS). The
RASS-SDSS galaxy cluster catalog is the first catalog which combines X-ray and optical data for a large number (114) of galaxy clusters. The systems are X-ray selected, ranging from groups of $10^{12.5} M_{\odot}$ to massive clusters of $10^{15} M_{\odot}$ in a redshift range from 0.002 to 0.45 . The X-ray (luminosity in the 
Table 6. The table presents the results of the best fit for the $i$ and $z$ bands in the region of minimum scatter, $0.2 \leq r \leq 1.0 \mathrm{Mpc} h_{70}^{-1}$. We show the results for the two methods applied: the orthogonal distance regression (ODRPACK) and the bisector method, which is the line bisecting the best fit results of the vertical and horizontal minimization. The $\alpha$ and $\beta$ parameters are given for several apertures with 95\% confidence errors. The orthogonal scatter and the scatters in $\log \left(T_{\mathrm{X}}\right)$ and in $\log \left(L_{\mathrm{op}}\right)$ to the best fit line are given by $\sigma, \sigma_{T_{\mathrm{X}}}$, and $\sigma_{L_{\mathrm{op}}}$, respectively.

\begin{tabular}{|c|c|c|c|c|c|c|c|c|c|c|}
\hline \multicolumn{11}{|c|}{$L_{\mathrm{op}}-T_{\mathrm{X}}$ relation in the $I$ band } \\
\hline \multicolumn{6}{|c|}{ Orthogonal method } & \multicolumn{5}{|c|}{ Bisector method } \\
\hline$r$ & $\alpha$ & $\beta$ & $\sigma$ & $\sigma_{T_{\mathrm{X}}}$ & $\sigma_{L_{\mathrm{op}}}$ & $\alpha$ & $\beta$ & $\sigma$ & $\sigma_{T_{\mathrm{X}}}$ & $\sigma_{L_{\mathrm{op}}}$ \\
\hline 0.2 & $0.97 \pm 0.17$ & $11.05 \pm 0.12$ & 0.53 & 0.39 & 0.36 & $1.22 \pm 0.22$ & $10.80 \pm 0.16$ & 0.54 & 0.39 & 0.37 \\
\hline 0.3 & $1.06 \pm 0.09$ & $11.21 \pm 0.06$ & 0.32 & 0.27 & 0.17 & $1.17 \pm 0.18$ & $11.12 \pm 0.14$ & 0.31 & 0.18 & 0.25 \\
\hline 0.5 & $1.11 \pm 0.08$ & $11.33 \pm 0.06$ & 0.26 & 0.20 & 0.16 & $1.13 \pm 0.10$ & $11.30 \pm 0.08$ & 0.25 & 0.17 & 0.19 \\
\hline 0.7 & $1.20 \pm 0.11$ & $11.43 \pm 0.08$ & 0.30 & 0.23 & 0.19 & $1.40 \pm 0.12$ & $11.27 \pm 0.09$ & 0.29 & 0.20 & 0.21 \\
\hline 0.8 & $1.20 \pm 0.12$ & $11.51 \pm 0.09$ & 0.33 & 0.26 & 0.20 & $1.51 \pm 0.13$ & $11.26 \pm 0.10$ & 0.33 & 0.23 & 0.24 \\
\hline 1.0 & $1.21 \pm 0.14$ & $11.60 \pm 0.10$ & 0.39 & 0.32 & 0.23 & $1.62 \pm 0.16$ & $11.26 \pm 0.12$ & 0.39 & 0.27 & 0.28 \\
\hline \multicolumn{11}{|c|}{$L_{\mathrm{op}}-T_{\mathrm{X}}$ relation in the $Z$ band } \\
\hline \multicolumn{6}{|c|}{ Orthogonal method } & \multicolumn{5}{|c|}{ Bisector method } \\
\hline$r$ & $\alpha$ & $\beta$ & $\sigma$ & $\sigma_{T_{\mathrm{X}}}$ & $\sigma_{L_{\mathrm{op}}}$ & $\alpha$ & $\beta$ & $\sigma$ & $\sigma_{T_{\mathrm{X}}}$ & $\sigma_{L_{\mathrm{op}}}$ \\
\hline 0.2 & $0.97 \pm 0.18$ & $11.18 \pm 0.13$ & 0.54 & 0.37 & 0.40 & $1.16 \pm 0.18$ & $10.95 \pm 0.13$ & 0.55 & 0.42 & 0.35 \\
\hline 0.3 & $1.06 \pm 0.08$ & $11.31 \pm 0.06$ & 0.31 & 0.27 & 0.16 & $1.18 \pm 0.19$ & $11.20 \pm 0.15$ & 0.31 & 0.18 & 0.25 \\
\hline 0.5 & $1.12 \pm 0.08$ & $11.42 \pm 0.06$ & 0.25 & 0.20 & 0.16 & $1.12 \pm 0.10$ & $11.39 \pm 0.08$ & 0.25 & 0.16 & 0.19 \\
\hline 0.7 & $1.20 \pm 0.11$ & $11.52 \pm 0.08$ & 0.30 & 0.24 & 0.19 & $1.40 \pm 0.12$ & $11.34 \pm 0.09$ & 0.30 & 0.20 & 0.23 \\
\hline 0.8 & $1.21 \pm 0.12$ & $11.60 \pm 0.09$ & 0.34 & 0.27 & 0.20 & $1.51 \pm 0.13$ & $11.34 \pm 0.10$ & 0.33 & 0.23 & 0.24 \\
\hline 1.0 & $1.22 \pm 0.14$ & $11.68 \pm 0.10$ & 0.39 & 0.32 & 0.23 & $1.62 \pm 0.15$ & $11.34 \pm 0.11$ & 0.39 & 0.27 & 0.28 \\
\hline
\end{tabular}

Table 7. The table presents the results of the best fit for the $i$ and $z$ bands in the region of minimum scatter, $0.2 \leq r \leq 1.0 \mathrm{Mpc} h_{70}^{-1}$ for the $L_{\mathrm{op}}-L_{\mathrm{X}}(R O S A T)$ relation in the subsample of REFLEX and NORAS X-ray selected clusters. We show the results for the orthogonal distance regression (ODRPACK) method. The $\alpha$ and $\beta$ parameters are given for several apertures with $95 \%$ confidence errors. The orthogonal scatter and the scatters in $\log \left(L_{\mathrm{X}}(0.1-2.4 \mathrm{keV})\right)$ and in $\log \left(L_{\mathrm{op}}\right)$ to the best fit line are given by $\sigma, \sigma_{L_{\mathrm{X}}}$, and $\sigma_{L_{\mathrm{op}}}$, respectively.

\begin{tabular}{c|ccccc|ccccc}
\hline \hline \multicolumn{10}{c}{$L_{\text {op }}-L_{\mathrm{X}}($ ROS AT $)$ relation for REFLEX-NORAS clusters only } \\
\hline \multicolumn{10}{c}{ Orthogonal method } \\
\hline \multicolumn{10}{c}{$i$ band } & \multicolumn{10}{c}{$z$ band } \\
\hline$r$ & $\alpha$ & $\sigma$ & $\sigma_{L_{X}}$ & $\sigma_{L_{\mathrm{op}}}$ & $\alpha$ & $\beta$ & $\sigma$ & $\sigma_{L_{\mathrm{X}}}$ & $\sigma_{L_{\mathrm{op}}}$ \\
\hline 0.2 & $0.28 \pm 0.04$ & $11.48 \pm 0.03$ & 0.32 & 0.23 & 0.22 & $0.27 \pm 0.04$ & $11.57 \pm 0.03$ & 0.31 & 0.23 & 0.21 \\
0.3 & $0.33 \pm 0.03$ & $11.74 \pm 0.02$ & 0.23 & 0.16 & 0.15 & $0.34 \pm 0.03$ & $11.83 \pm 0.02$ & 0.21 & 0.16 & 0.15 \\
0.5 & $0.36 \pm 0.03$ & $11.87 \pm 0.02$ & 0.22 & 0.16 & 0.15 & $0.36 \pm 0.03$ & $11.97 \pm 0.02$ & 0.22 & 0.16 & 0.14 \\
0.7 & $0.36 \pm 0.03$ & $12.01 \pm 0.02$ & 0.24 & 0.18 & 0.16 & $0.37 \pm 0.03$ & $12.11 \pm 0.02$ & 0.24 & 0.17 & 0.16 \\
0.8 & $0.37 \pm 0.03$ & $12.08 \pm 0.03$ & 0.27 & 0.20 & 0.17 & $0.37 \pm 0.03$ & $12.17 \pm 0.02$ & 0.26 & 0.20 & 0.17 \\
1.0 & $0.37 \pm 0.04$ & $12.18 \pm 0.03$ & 0.29 & 0.23 & 0.18 & $0.37 \pm 0.04$ & $12.28 \pm 0.03$ & 0.28 & 0.22 & 0.18 \\
\hline
\end{tabular}

ROSAT band, bolometric luminosity, redshift, center coordinates) and optical properties (Schechter luminosity function parameters, luminosity, central galaxy density, core, total and half-light radii) are computed in a uniform and accurate way. The catalog also contains temperatures and iron abundances for a subsample of 53 clusters from the Asca Cluster Catalog and the Group Sample. The resulting RASS-SDSS galaxy cluster catalog constitutes an important database to study the properties of galaxy clusters and in particular the relation of the galaxy population seen in the optical to the properties of the $\mathrm{X}$-ray luminous ICM.

The first investigations reported have shown a tight correlation between the X-ray and optical properties, when the choice of the measurement aperture for the optical luminosity and the optical wavelength band are optimized. We find that the optical luminosity calculated in the $i$ and in the $z$ band correlates better with the X-ray luminosity and the ICM temperature, than is the case for the other Sloan photometric bands. Thus the red optical bands, which are more sensitive to the light of the old stellar population and therefore to the stellar mass of cluster galaxies, have tight correlations with the X-ray properties of the systems.

Moreover, we found that the scatter in the $L_{\mathrm{op}}-L_{\mathrm{X}}$ and $L_{\mathrm{op}}-T_{\mathrm{X}}$ relations can be minimized if the optical luminosity is measured within a cluster aperture between $0.2-0.8 \mathrm{Mpc} h_{70}^{-1}$, with an absolute minimum of the scatter at $0.5 \mathrm{Mpc} h_{70}^{-1}$. The best aperture for the measurement of the optical luminosity is due to the fact that it is a good compromise for assessing simultaneously the total richness and the compactness of the cluster. 
Finally, using the relations obtained from the $z$ band, we demonstrated that, given the optical properties of a cluster, we can predict the X-ray luminosity and temperature with an accuracy of $60 \%$ and vice versa. By restricting the correlation analysis to the subsample of X-ray detected REFLEX-NORAS clusters, the minimum scatter decreases to less than $40 \%$ for the $L_{\mathrm{op}}-L_{\mathrm{X}}$ relation. Since the observational uncertainties in the optical and in the X-ray luminosity are about $20 \%$, the observed scatter in both relations should be intrinsic.

The resulting logarithmic slope for the $L_{\mathrm{op}}-L_{\mathrm{X}}$ relation with the minimum scatter is $0.38 \pm 0.02$, while the value for the $L_{\mathrm{op}}-T_{\mathrm{X}}$ relation is $1.12 \pm 0.08$. These results are not consistent with the assumption of hydrostatic equilibrium and constant $M / L$. If we assume that $M / L$ depends on the luminosity with a power law $M / L \propto L^{0.3}$ (Girardi et al. 2002), our results are in very good agreement with the expected values under the assumption of hydrostatic equilibrium.

The analysis carried out in this paper on the correlation between X-ray and optical appearance of galaxy clusters is completely empirical. In principle, the best way to proceed in this kind of study is to measure the optical luminosity within the physical size of the cluster, i.e. whitin the virial radius. Without optical spectroscopic data or accurate temperature measurements, the cluster virial radius can be calculated assuming a theoretical model relating the optical luminosity to the cluster mass. At this stage of the work, we preferred to tackle the cluster X-ray-optical connection with the empirical method explained in the paper, in order to have model-independent results. On the other hand, not taking into account the different cluster sizes could have affected both the slope and the scatter of the given relations (Eqs. (14)-(16)). Therefore, for a better understanding of the important connection between the X-ray and optical appearance of galaxy clusters, the optical luminosity has to be calculated within the physical size of the cluster. This work is in progress and will be published in the second paper of this series on the RASS-SDSS galaxy cluster sample. The next step will be the study of the fundamental plane of galaxy clusters. Through this kind of analysis we will find out if the observed scatter in the correlations between the optical and X-ray properties depends on another parameter related to the cluster compactness. Moreover, because of the link between the galaxy cluster fundamental plane and the $M / L$ parameter, we will connect directly the slope of two relations to the behavior of $M / L$.

Acknowledgements. Funding for the creation and distribution of the SDSS Archive has been provided by the Alfred P. Sloan Foundation, the Participating Institutions, the National Aeronautics and Space Administration, the National Science Foundation, the US Department of Energy, the Japanese Monbukagakusho, and the Max Planck Society. The SDSS Web site is http://www.sdss.org/. The SDSS is managed by the Astrophysical Research Consortium (ARC) for the Participating Institutions. The Participating Institutions are The University of Chicago, Fermilab, the Institute for Advanced Study, the Japan Participation Group, The Johns Hopkins University, Los Alamos National Laboratory, the Max-PlanckInstitute for Astronomy (MPIA), the Max-Planck-Institute for Astrophysics (MPA), New Mexico State University, University of
Pittsburgh, Princeton University, the United States Naval Observatory, and the University of Washington.

\section{References}

Abazajian, K., Adelman, J., Agueros, M., et al. 2003, AJ, 126, 2081 (Data Release One)

Adami, C., Mazure, A., Katgert, P., et al. 1998, A\&A, 336, 63

Akritas, M., Mazure, A., Katgert, P., et al. 1996, ApJ, 470, 706

Arnaud, M., Rothenflug, R., Boulade, O., et al. 1992, A\&A, 254, 49

Bahcall, N. 1977, ApJ, 217, L77

Bahcall, N. 1981, ApJ, 247, 787

Bahcall, N., McKay, T. A., Annis, J., et al. 2003, ApJS, 148, 253

Blanton, M. R., Dalcanton, J., Eisenstein, D., et al. 2001, AJ, 121, 2358

Blanton, M. R., Lupton, R. H., Maley, F. M., et al. 2003, AJ, 125, 2276 (Tiling Algorithm)

Böhringer, H., Voges, W., Huchra, J. P., et al. 2000, ApJS, 129, 435

Böhringer, H., Schuecker, P., Guzzo, L., et al. 2001, A\&A, 369, 826

Böhringer, H., Collins, C. A., Guzzo, L., et al. 2002, ApJ, 566, 93

De Grandi, S., \& Molendi, S. 2002, ApJ, 567, 163

De Propris, R., Colless, M., Driver, S. P., et al. 2003, MNRAS, 342, 725

Donahue, M., Mack, J., Scharf, C., et al. 2001, ApJ, 552, L93

Donahue, M., Scharf, C. A., Mack, J., et al. 2002, ApJ, 569, 689

Dressler, A., Oemler, A. Jr., Couch, W. J., et al. 1997, ApJ, 490, 577

Edge, A. C., \& Stewart, G. C. 1991, MNRAS, 252, 428

Eisenstein, D. J., Annis, J., Gunn, J. E., et al. 2001, AJ, 122, 2267

Fasano, G., Poggianti, B. M., Couch, W. J., et al. 2000, ApJ, 542, 673

Finoguenov, A., Arnaud, M., \& David, L. P. 2001, ApJ, 555, 191F

Finoguenov, A., Reiprich, T. H., \& Böhringer, H. 2001, A\&A, 368, 749

Fletcher, R. 1970, Comput. J., 13, 317

Fukugita, M., Shimasaku, K., \& Ichikawa, T. 1995, PASJ, 107, 945

Fukugita, M., Ichikawa, T., \& Gunn, J. E. 1996, AJ, 111, 1748

Girardi, M., Borgani, S., Giuricin, G., et al. 2000, ApJ, 530, 62

Girardi, M., Manzato, P., Mezzetti, M., et al. 2002, ApJ, 569, 720

Gladders, M., \& Yee, H. K. C. 2000, AJ, 120, 2148

Gomez, P., Nichol, R. C., Miller, C. J., et al. 2003, ApJ, 584, 210

Goto, T., Sekiguchi, M., Nichol, R. C., et al. 2002a, AJ, 123, 1807

Goto, T., Okamura, S., McKay, T. A., et al. 2002b, PASP, 123, 1807

Gunn, J. E., Carr, M. A., Rockosi, C. M., et al. 1998, AJ, 116, 3040 (SDSS Camera)

Hogg, D. W., Finkbeiner, D. P., Schlegel, D. J., \& Gunn, J. E. 2001, AJ, 122, 2129

Horner, D. 2001, Ph.D. Thesis, University of Maryland

Ikebe, Y., Reiprich, T. H., Böhringer, H., et al. 2002, A\&A, 383, 773

Isobe, T., Feigelson, E. D., Akritas, M. G., et al. 1990, ApJ, 364, 104 Kelson, D., van Dokkum, P. G., Franx, M., et al. 1997, ApJ, 478, 13

Kelson, D., Illingworth, G. D., van Dokkum, P. G., \& Franx, M. 2000, ApJ, 531, 137

Kim, R., Seung, J., \& Kepner, J. V. 2002, AJ, 123, 20

Kochanek, C. S., Pahre, M. A., Falco, E. E., et al. 2001, ApJ, 560, 566

Lin, Y., Mohr, J. J., \& Stanford, S. A. 2003, ApJ, 591, 749

Lubin, L., Oke, J. B., \& Postman, M. 2002, AJ, 124, 1905

Lumsden, S. L., Collins, C. A., Nichol, R. C., et al. 1997, MNRAS, 290, 119

Lupton, R. H., Gunn, J. E., \& Szalay, A. S. 1999, AJ, 118, 1406

Lupton, R., Gunn, J. E., Ivezić, Z., et al. 2001, in Astronomical data analysis software and systems X, ed. F. R. Harnden, Jr., F. A. Primini, \& H. E. Payne (San Francisco: Astr. Soc. Pac.), ASP Conf. Ser., 238, 269 [arXiv: astro-ph/0101420]

Markevitch, M. 1998, ApJ, 504, 27 
Mulchaey, J. S., Davis, D. S., Mushotzky, R. F., \& Burstein, D. 2003, ApJSS, 145, 39

Pier, J. R., Munn, J. A., Hindsley, R. B., et al. 2003, AJ, 125, 1559 (Astrometry)

Poggianti, B., Bridges, T. J., Komiyama, Y., et al. 2004, ApJ, 601, 197 Postman, M., Lubin, L. M., \& Oke, J. B. 1998, AJ, 116, 560

Reiprich, T. H., \& Böringher, H. 2002, ApJ, 567, 716

Retzlaff, J. 2001, XXIst Moriond Astrophysics Meeting, March 10-17, 2001 Savoie, France, ed. D. M. Neumann, \& J. T. T. Van

Rosati, P., Borgani, S., \& Norman, C. 2002, ARA\&A, 40, 539

Sarazin, C. 1980, ApJ, 236, 75

Schlegel, D., Finkbeiner, D. P., \& Davis, M. 1998, ApJ, 500, 525
Shimasaku, K., Fukugita, M., Doi, M., et al. 2001, AJ, 122, 1238

Smith, J. A., Tucker, D. L., Kent, S. M., et al. 2002, AJ, 123, 2121

Stoughton, C., Lupton, R. H., Bernardi, M., et al. 2002, AJ, 123, 485

Strateva, I., Ivezić, Z., Knapp, G. R., et al. 2001, AJ, 122, 1861

Strauss, M. A., Weinberg, D. H., Lupton, R. H., et al. 2002, AJ, 124, 1810

van Dokkum, S., Franx, M., Fabricant, D., et al. 2000, ApJ, 541, 95V

Voges, W., Aschenbach, B., Boller, Th., et al. 1999, A\&A, 349, 389

Xue, Y., \& Wu, X. 2000, ApJ, 538, 65

Yasuda, N., Fukugita, M., Narayanan, V. K., et al. 2001, AJ, 122, 1104

Yee, H. K. C., \& Ellingson, E. 2003, ApJ, 585, 215

York, D. G., Adelman, J., Anderson, J. E., et al. 2000, AJ, 120, 1579

Ziegler, B., \& Bender, R. 1997, MNRAS, 291, 527 